\title{
Renormalization schemes and the range of two-nucleon effective field theory
}

\author{
Thomas Mehen and Iain W. Stewart \\ California Institute of Technology, Pasadena, California 91125
}

(Received 20 October 1998)

\begin{abstract}
The off-shell momentum subtraction (OS) and power divergence subtraction (PDS) renormalization schemes for the effective field theory with nucleons and pions are investigated. We explain in detail how the renormalization is implemented using local counterterms. Fits to the $N N$ scattering data are performed in the ${ }^{1} S_{0}$ and ${ }^{3} S_{1}$ channels for different values of $\mu_{R}$. An error analysis indicates that the range of the theory with perturbative pions is consistent with $500 \mathrm{MeV}$. [S0556-2813(99)05305-4]
\end{abstract}

PACS number(s): 13.75.Cs, 11.10.Gh, 21.30.-x, 24.85.+p

\section{INTRODUCTION}

Effective field theory is an important tool for studying nuclear interactions. To describe low energy processes involving nucleons and pions in a model-independent way, all possible operators consistent with the symmetries of QCD are included in an effective Lagrangian. The short distance strong interaction physics is parametrized by contact interactions, which can be thought of as arising from massive states which have been integrated out. The real power of effective field theory is that theoretical errors can be estimated in a systematic way. Different contributions to an observable are organized by a power counting. This means we have a way of organizing the theory as an expansion in $Q / \Lambda$, where $Q$ is a momentum scale which characterizes the process under consideration and $\Lambda$ is the range of validity of the effective theory. To a given order in $Q / \Lambda$, only a finite number of operators in the effective Lagrangian need be retained, and observables can be predicted in terms of a finite number of experimentally determined parameters. Theoretical uncertainty in the calculation can be reliably estimated and reduced by calculating higher orders in $Q / \Lambda$. Though $\Lambda$ is not known a priori, it is expected to be set by the masses of the lightest particles which have not been explicitly included in the effective Lagrangian.

In an effective field theory, ultraviolet divergences must be regulated and a renormalization scheme defined. The ultraviolet divergences give a constraint on the power counting since when a divergent loop graph occurs one must include a contact operator that can absorb the divergence at the same or lower order in $Q$. This is familiar from pion chiral perturbation theory. The choice of regulator cannot affect physical results, but may make implementing a renormalization scheme easier. The renormalization scheme and power counting are also tied together. In a natural scheme, the renormalized coefficients of the operators in the Lagrangian are normal in size based on dimensional analysis with $\Lambda$. Once a power counting is established one can translate between different renormalization schemes at a given order in $Q$ without changing the physical predictions.

Counting powers of $Q / \Lambda$ in the nuclear effective theory is a subtle issue because of the large $S$-wave scattering length $a$. Usually in an effective theory (e.g., chiral perturbation theory for pions), the coefficients of operators of dimension $n+4$ are assumed to scale as $\Lambda^{-n}$. It is then straightforward to examine an arbitrary graph and determine its power in $Q / \Lambda$. Applying this approach to the nucleon-nucleon interaction does not work because the large scattering lengths introduce an unnatural length scale. Since $1 / a\left({ }^{1} S_{0}\right)=$ $-8.32 \mathrm{MeV}$ and $1 / a\left({ }^{3} S_{1}\right)=36.4 \mathrm{MeV}$, it is necessary to sum corrections that scale like $(Q a)^{n}$ to all orders. Therefore, the power counting must be modified. A detailed discussion of power counting in the presence of a large scattering length is given in Refs. [1-4]. In Ref. [1], it is pointed out that the large scattering length changes the power counting for graphs with intermediate nucleons and four-nucleon couplings with no derivatives. In Refs. [2,3], Kaplan, Savage, and Wise (KSW) point out that the effects of the large scattering length can be incorporated into the theory by assigning a power counting to the coefficients of four-nucleon operators. The power counting for coefficients of operators mediating $S$-wave transitions is changed, as well as other coefficients because of angular momentum mixing. A more detailed discussion of this power counting is left to the next section. A similar power counting is discussed in Ref. [4].

Two different calculational techniques for the effective theory of nucleons are used in the literature. In one approach, the power counting is applied to regulated $N$-nucleon potentials and the Schrödinger equation is solved [1,5-7]. Solving the Schrödinger equation is equivalent to including all ladder graphs with the potential as the two-particle irreducible kernel (see, e.g., [8]). The second approach, advocated by KSW, is like ordinary chiral perturbation theory in that the power counting is applied directly to the Feynman graphs which contribute to the amplitude. Here the method for dealing with nuclear bound states and infrared divergences that occur at zero kinetic energy is similar to the methods used in nonrelativistic QED and QCD [9]. A nonrelativistic propagator is used which includes the kinetic energy term to regulate the infrared divergence. In the Feynman diagram approach, dimensional regularization is the most convenient regulator, and analytic results are readily obtained. In the potential method, the Schrödinger equation is usually solved numerically. In practice, divergences are regulated and renormalized couplings are defined using a finite cutoff scheme. In Ref. [10], it has been explicitly shown that without pions the potential method can deal with large scattering lengths, and gives an expansion in $Q / \Lambda$. 


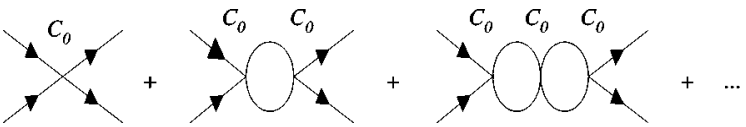

FIG. 1. The leading order contribution to $N N$ scattering in the KSW power counting.

An important aspect of the KSW analysis is the use of a novel renormalization scheme, power divergence subtraction (PDS). In the PDS scheme, loop integrals in Feynman graphs are regulated using dimensional regularization, and poles in both $d=3$ and $d=4$ are subtracted. The subtraction of $d$ $=3$ poles gives a power law dependence on the renormalization point $\mu_{R}$ to the coefficients of four-nucleon operators. Let us use $C_{2 m}$ to denote the coefficient of a four-nucleon operator with $2 \mathrm{~m}$ derivatives (where, for the moment, we restrict ourselves to operators mediating $S$-wave transitions). Choosing $\mu_{R} \sim Q$, graphs with an arbitrary number of $C_{0}\left(\mu_{R}\right)$ vertices scale as $1 / Q$ and must be summed to all orders, as shown in Fig. 1. This is precisely the set of graphs that sums corrections that scale like $(Q a)^{n}$. Higher order contributions form a series in $Q / \Lambda$. In Ref. [4], it is emphasized that it is possible to phrase the power counting in a scheme-independent manner. The choice of scheme is simply to give natural-sized coefficients which make the power counting manifest, i.e., $C_{2 m}\left(\mu_{R}\right) \sim 4 \pi /\left(M \Lambda^{m} \mu_{R}^{m+1}\right)$, where $C_{2 m}\left(\mu_{R}\right)$ are the renormalized couplings. PDS is one example of such a scheme. In Ref. [11], it is shown how the KSW power counting can be implemented by solving the Schrödinger equation in a finite cutoff scheme.

Pions can be added to the effective field theory by identifying them as the pseudo-Goldstone bosons of the spontaneously broken chiral symmetry of QCD. All operators with the correct transformation properties are added to the effective Lagrangian. This includes operators with insertions of the light quark mass matrix and derivatives, whose coefficients are needed to cancel ultraviolet divergences from loop graphs. In dimensional regularization, these ultraviolet divergences are of the form $p^{2 n} m_{\pi}^{2 m} / \epsilon$. For instance, for nucleons in the ${ }^{3} S_{1}$ channel, the two-loop graph with three pions and a two-loop graph with two pions and one $C_{0}$ have ultraviolet divergences of the form $p^{2} / \epsilon$. This pole must be canceled by a counterterm involving a four-nucleon operator with two derivatives. Because divergences of the form $p^{2 n} / \epsilon$ must be canceled by local counterterms, pion exchange can only be calculated in a model-independent way if higher derivative contact interactions are included at the same order that these divergences occur [12,13]. In Weinberg's [1] power counting, pion exchange is included in the leading order potential. Therefore, graphs with arbitrary numbers of pions are leading order, while the counterterms necessary to cancel the ultraviolet divergences in these graphs are subleading. However, the potential method can still be used. As higher order derivative operators are added to the potential the accuracy is systematically improved, because the onset of the model dependence of the pion summation appears at higher order in $Q / \Lambda$. For example, the cutoff dependence of the two-pion graph with one $C_{0}$ will be canceled by the cutoff dependence in $C_{2}$.

Different estimates of the range $\Lambda_{\pi}$ of an effective theory of nucleons with perturbative pions exist in the literature.
Some authors [14-16] argue that $\Lambda_{\pi}$ is as small as $m_{\pi}$, so that including perturbative pions is superfluous. One estimate of the range is given by KSW who conclude that $\Lambda_{\pi}$ $\sim 300 \mathrm{MeV}$. They point out that in PDS the renormalization group equation for the coefficient $C_{0}\left(\mu_{R}\right)$ is modified by the inclusion of pions in such a way that for $\mu_{R} \gtrsim 300 \mathrm{MeV}$, $C_{0}\left(\mu_{R}\right)$ scales like $\mu_{R}^{0}$ instead of $\mu_{R}^{-1}$. Since the power counting is no longer manifest above this scale, KSW conclude that the effective theory breaks down at this point. In Ref. [15] different renormalized couplings are obtained. Here a breakdown of the power counting for $C_{2}\left(\mu_{R}\right)$ at $\mu_{R} \sim m_{\pi}$ is observed. A crucial question is whether a breakdown in the running of the coupling constants is a physical effect or simply an artifact of the renormalization scheme. It is dangerous to draw conclusions based on the large momentum behavior of the coupling constants because the beta functions of the couplings are scheme dependent. ${ }^{1}$ In Ref. [17], a momentum subtraction scheme is introduced where the power law dependence of the coupling constants persists even in the presence of pions and for all values of $\mu_{R}>1 / a$. This scheme is called the OS scheme, since in a relativistic theory it might be called an off-shell momentum subtraction scheme. In Ref. [18], a similar scheme is applied to the spin-singlet channel in the theory without pions, where it is shown to give results identical to the PDS scheme. The OS scheme is a natural scheme that works with arbitrary partial waves and with pions. Thus, the range of the validity of the effective theory is not limited by the large $\mu_{R}$ behavior of the couplings. The PDS scheme is still useful for calculating observables. If one splits $C_{0}\left(\mu_{R}\right)$ into a nonperturbative and perturbative part, $C_{0}\left(\mu_{R}\right)=C_{0}^{\mathrm{p}}\left(\mu_{R}\right)+C_{0}^{\mathrm{np}}\left(\mu_{R}\right)$, then $C_{0}^{\mathrm{np}}\left(\mu_{R}\right) \sim 1 / \mu_{R}$ for all $\mu_{R}>1 / a$. Once this split has been performed, it is straightforward to establish relations between the OS and PDS schemes order by order in perturbation theory, and any prediction for an observable will be identical in the two schemes up to the order in $Q / \Lambda_{\pi}$ to which it is calculated. Since in both schemes there is no scale where the power counting breaks down, it is possible that $\Lambda_{\pi}>300 \mathrm{MeV}$.

Physically, one expects the effective theory to be valid up to a threshold where new degrees of freedom can be created on shell. For elastic nucleon scattering, the relevant physical threshold is production of $\Delta$ resonances which occurs at $p$ $=\sqrt{M_{N}\left(M_{\Delta}-M_{N}\right)}=525 \mathrm{MeV}$ [the $S$-wave channels couple only to the $\Delta \Delta$ intermediate state, and so $p$ $\left.=\sqrt{2 M_{N}\left(M_{\Delta}-M_{N}\right)}=740 \mathrm{MeV}[19]\right]$. Above this scale, the $\Delta$ must be included as an explicit degree of freedom. Below this scale, the $\Delta$ can be integrated out, leaving an effective theory of pions and nucleons. Rho exchange becomes relevant at a scale $p \sim m_{\rho}=770 \mathrm{MeV}$. There is also a $N^{*}(1440) N$ intermediate state with a threshold of $p$ $=685 \mathrm{MeV}$. One might expect $\Lambda_{\pi}$ to be of the order of these thresholds. However, there is an intermediate scale of $300 \mathrm{MeV}$ associated with short distance contributions from

\footnotetext{
${ }^{1}$ This is in contrast with dimensionless coupling constants like $g$ in QCD. In that case the first two coefficients of the beta function are scheme independent; so conclusions based on the behavior of the running coupling constant at small coupling (e.g., asymptotic freedom) are physical.
} 
potential pion exchange. ${ }^{2}$ Using dimensional analysis, a graph with the exchange of $n+1$ potential pions is suppressed by $p / 300 \mathrm{MeV}$ relative to a graph with $n$ potential pions. Comparison of the size of individual graphs is scheme dependent [for example, the size of graphs differs in the minimal subtraction (MS) and modified minimal subtraction $(\overline{\mathrm{MS}})$ schemes]. The $300 \mathrm{MeV}$ scale applies only to a subset of graphs, and may change once all graphs at a given order in $Q$ are included in the estimate. Therefore, $300 \mathrm{MeV}$ can be taken as an order of magnitude estimate for the range of the theory, but the actual range may be enhanced or suppressed by an additional numerical factor.

This then motivates the important question: How does one determine the range of the effective field theory? This is obviously a question of great practical importance. Theoretical arguments can only give an approximate estimate for the range. A good example comes from SU(3) chiral perturbation theory. In this strong coupling theory, it is natural to expect that the range of the theory is the chiral symmetry breaking scale $\Lambda_{\chi} \sim 2 \sqrt{2} f_{\pi}=1200 \mathrm{MeV}$ [20]. However, the convergence of the momentum expansion will depend on the particular process under consideration. For instance, in $\pi-\pi$ scattering the range of the expansion is set by the threshold for $\rho$ production, $m_{\rho}=770 \mathrm{MeV}$. In this paper, the range of the two-nucleon effective theory will be estimated using nucleon-nucleon scattering data. Our results are consistent with $\Lambda_{\pi} \sim 500 \mathrm{MeV}$. As we will explain in Sec. VI, the error analysis is applied to $\delta$ rather than to $p \cot \delta$ as in Ref. [15]. This range does not depend on the value of the renormalization point chosen, and is found in both the OS and PDS schemes. However, only next-to-leading order calculations have been used, and so it is hard to estimate the error in this value. When higher order corrections are computed, it should be possible to obtain a reasonably accurate estimate of the range of the two-nucleon effective field theory with perturbative pions. This $500 \mathrm{MeV}$ estimate is based solely on phase shift data. Predictions for the deuteron electromagnetic form factors are also in reasonable agreement with the data [21], but do not probe momenta greater than $200 \mathrm{MeV}$, and so do not provide interesting information regarding the range of the theory.

In Sec. II, we review the power counting method of KSW $[2,3]$ and the PDS scheme. The importance of being able to count factors of the large nucleon mass in a nonrelativistic effective field theory is discussed. We review the OS scheme, which is compatible with the KSW power counting. We describe the procedure for defining the renormalized couplings using local counterterms for each of these schemes.

In Sec. III, we discuss the theory with only nucleons, where $\Lambda \sim m_{\pi}$. Local counterterms for both the PDS and OS schemes are computed. These counterterms are used to obtain the beta functions for the four-nucleon operators, and we explain why the beta functions for the most relevant operators in this theory are one-loop exact.

\footnotetext{
${ }^{2}$ The phrase "potential pion exchange" will be used for a perturbative pion with energy-independent propagator. This is sometimes called static pion exchange.
}

The theory with nucleons and pions is analyzed in Sec. IV. In the ${ }^{3} S_{1}$ channel, there are corrections to the PDS beta functions at all orders in $Q$. As examples, we compute the PDS beta functions for $C_{0}^{\left({ }^{3} S_{1}\right)}\left(\mu_{R}\right)$ to order $Q$ and for $C_{2}^{\left({ }^{3} S_{1}\right)}\left(\mu_{R}\right)$ to order $Q^{0}$. In this channel, even in the limit $m_{\pi} \rightarrow 0$, there are logarithmic divergences (poles of the form $p^{2} / \epsilon$ in dimensional regularization). In the OS scheme, the ${ }^{3} S_{1}$ beta functions can be calculated exactly. We compute the exact beta functions for $C_{0}\left(\mu_{R}\right), C_{2}\left(\mu_{R}\right)$, and $C_{4}\left(\mu_{R}\right)$ in the OS scheme in the ${ }^{1} S_{0}$ and ${ }^{3} S_{1}$ channels. In Sec. V, the counterterms for the coupling constant $D_{2}\left(\mu_{R}\right)$ are derived in the OS and PDS schemes.

In Sec. VI, we discuss why it is important to have $\mu_{R}$ independent amplitudes order by order in the expansion. In the OS scheme amplitudes are $\mu_{R}$ independent, while in PDS $\mu_{R}$ independent amplitudes can be obtained by treating part of $C_{0}\left(\mu_{R}\right)$ perturbatively. If this is not done, then the sensitivity to $\mu_{R}$ is larger than one might expect [14], for reasons we explain. Fits to the data are presented for different values of $\mu_{R}$ and the coupling constants in both the OS and PDS schemes are shown to evolve according to the renormalization group equations.

In Sec. VII, an error analysis similar to a method due to Lepage [6] is used to investigate the range of the effective field theory with perturbative pions at next-to-leading order. Weighted fits are performed for the scattering data in both the ${ }^{1} S_{0}$ and ${ }^{3} S_{1}$ channels. Our results rule out $\Lambda_{\pi} \sim m_{\pi}$ and are consistent with $\Lambda_{\pi} \sim 500 \mathrm{MeV}$.

\section{POWER COUNTING AND RENORMALIZATION SCHEMES}

In this section, the KSW power counting and compatible renormalization schemes are discussed. The theory containing only nucleon fields is considered first. Some notation is set, and we explain why the large nucleon mass does not affect the power counting. The renormalized couplings are then defined in terms of local counterterms, and the KSW power counting for coefficients of four-nucleon operators is reviewed. Next, we consider the theory including pions. We review the power counting for potential and radiation pions, and explain the origin of the $300 \mathrm{MeV}$ scale associated with potential pion exchange. The PDS renormalization scheme is then discussed and we introduce the OS momentum subtraction scheme, which is also compatible with the power counting.

Below the scale $m_{\pi}$, the pion can be integrated out, leaving a theory of nonrelativistic nucleons interacting via contact interactions. The Lagrangian in the two-nucleon sector is given by

$$
\mathcal{L}_{N N}=N^{\dagger}\left[i \partial_{t}+\vec{\nabla}^{2} /(2 M)+\cdots\right] N-\sum_{s} \sum_{m=0}^{\infty} C_{2 m}^{(s)} \mathcal{O}_{2 m}^{(s)},
$$

where $M$ is the nucleon mass, and the ellipsis refers to relativistic corrections. $\mathcal{O}_{2 m}^{(s)}$ is an operator with $2 m$ spatial derivatives and four-nucleon fields $N$. We will work in a basis in which the operators mediate transitions between ingoing and outgoing states of definite total angular momentum. Our 
notation is set up to agree with Refs. [2,3]. The superscript $s$ will give the angular momentum quantum numbers of these states in the standard spectroscopic notation ${ }^{2 S+1} L_{J}$. If we denote the incoming and outgoing orbital angular momenta by $L$ and $L^{\prime}$, then any operator mediating a transition between these states must contain at least $L+L^{\prime}$ derivatives. For states with $S=0,\left|L-L^{\prime}\right|=0$, while for states with $S$ $=1,\left|L-L^{\prime}\right|=0$ or 2 . For $L=L^{\prime}=0$ the first few terms in the series are

$$
\begin{aligned}
\sum_{s, m} C_{2 m}^{(s)} \mathcal{O}_{2 m}^{(s)}= & C_{0}^{(s)}\left(N^{T} P_{i}^{(s)} N\right)^{\dagger}\left(N^{T} P_{i}^{(s)} N\right) \\
& -\frac{C_{2}^{(s)}}{8}\left[\left(N^{T} P_{i}^{(s)} N\right)^{\dagger}\left(N^{T} P_{i}^{(s)} \stackrel{\leftrightarrow}{\nabla} N\right)+\text { H.c. }\right] \\
& +\cdots
\end{aligned}
$$

where the matrices $P_{i}^{(s)}$ project onto the correct spin and isospin states:

$$
P_{i}^{\left({ }^{1} S_{0}\right)}=\frac{1}{\sqrt{8}}\left(i \sigma_{2}\right)\left(i \tau_{2} \tau_{i}\right), \quad P_{i}^{\left({ }^{3} S_{1}\right)}=\frac{1}{\sqrt{8}}\left(i \sigma_{2} \sigma_{i}\right)\left(i \tau_{2}\right)
$$

The Galilean-invariant derivative in Eq. (2.2) is $\stackrel{\nabla}{ }^{2}=\overleftarrow{\nabla}^{2}$ $-2 \overleftarrow{\nabla} \cdot \vec{\nabla}+\vec{\nabla}^{2}$, and the ellipsis denotes contributions with more derivatives and/or higher partial waves.

The $C_{2 m}$ appearing in Eq. (2.1) are bare parameters. To renormalize the theory, the bare coupling is separated into a renormalized coupling and counterterms as follows:

$$
\begin{gathered}
C_{2 m}^{\text {bare }}=C_{2 m}^{\text {finite }}-\delta^{\mathrm{uv}} C_{2 m} \\
C_{2 m}^{\text {finite }}=C_{2 m}\left(\mu_{R}\right)-\sum_{n=0}^{\infty} \delta^{n} C_{2 m}\left(\mu_{R}\right) .
\end{gathered}
$$

Note that we divide the counterterms into two classes. The first, which have the superscript "uv," contain all genuine ultraviolet divergences. These include $1 / \epsilon$ poles, if dimensional regularization is used, or powers and logarithms of the cutoff if a hard cutoff is used. We will also include some finite constants [e.g., the $-\gamma+\ln (4 \pi)$ that is subtracted in the $\overline{\mathrm{MS}}$ scheme] if this proves to be convenient for keeping expressions compact. By construction, these counterterms are $\mu_{R}$ independent, but will depend on $C_{2 m}^{\text {finite }}$. The renormalized coupling is denoted $C_{2 m}\left(\mu_{R}\right)$. The remaining counterterms $\delta^{n} C_{2 m}\left(\mu_{R}\right)$, contain no ultraviolet divergences and will be referred to as the finite counterterms. The choice of the finite counterterms differentiates between the schemes in our paper. An infinite number of finite counterterms are needed because an infinite number of loop graphs are included at leading order. The renormalization is carried out order by order in the loop expansion. The superscript $n$ indicates that $\delta^{n} C_{2 m}$ is included at the tree level for a graph with $n$ loops. When higher loop graphs are considered, the $\delta^{n} C_{2 m}$ counterterm takes the place of $n$ loops [22]. For example, at three loops we have three-loop diagrams with renormalized couplings at the vertices, two-loop diagrams with a $\delta^{1} C$ counterterm, one-loop diagrams with either one $\delta^{2} C$ or two $\delta^{1} C^{\prime}$ 's, and a tree level diagram with $\delta^{3} C$. Examples are given in Sec. III and Appendix A.

For the nucleon theory, the kinematic part of the power counting is very simple $[1,21]$. $Q$ is identified with a typical external momentum characterizing the process under consideration. For instance, in elastic nucleon-nucleon scattering $Q \sim p$, where $p$ is the center-of-mass momentum. ${ }^{3}$ Each nucleon propagator gives a $Q^{-2}$, each spatial derivative a $Q$, each time derivative a $Q^{2}$, and each loop integration a $Q^{5}$.

For nonrelativistic nucleons, the scale $M$ is much larger than typical momenta. The reason for using a nonrelativistic expansion is that each graph will scale as a definite power of $M$. To see this, rescale all energies $q^{0} \rightarrow \widetilde{q}^{0} / M$ or, equivalently, time coordinates $t \rightarrow M \tilde{t}$, so that dimensionful quantities have the same size. Since the measure $d^{4} x \sim M$, the Lagrange density $\mathcal{L} \sim 1 / M$. In coordinate space our nucleon fields scale as $N(x) \sim M^{0}$ [in momentum space $\mathcal{L} \sim M$ and $N(p) \sim \sqrt{M}]$; so, from Eq. (2.1), $C_{2 m} \sim 1 / M$. With the $M$ scaling for the couplings determined we can find the scaling of any Feynman graph. A nucleon propagator gives one power of $M$, each momentum space loop integration a $1 / M$, and the $M$ 's from external lines are canceled by $M$ 's from the states. For graphs that have insertions of the four-nucleon operators $N_{P}=N_{L}+N_{V}-1$, where $N_{P}, N_{L}, N_{V}$ are the number of propagators, loops, and vertices. Thus, at leading order in the nonrelativistic expansion any graph built out of the interactions in Eq. (2.2) scales as $M^{-1}$ since $N_{P}-N_{L}-N_{V}$ $=-1$. Therefore, the $2 \rightarrow 2$ scattering amplitude $\mathcal{A} \sim 1 / M$. With the definition of $\mathcal{A}$ used here this scaling gives a finite cross section in the $M \rightarrow \infty$ limit which is physically sensible. Since all graphs scale the same way with $M, M$ is irrelevant to the power counting. Relativistic corrections are included perturbatively [3,23], and are generally suppressed by $Q^{2} / M^{2}$ relative to the leading contribution to an observable. This type of correction will not be considered here.

In the theory with only nucleons, the only graphs relevant to $2 \rightarrow 2$ scattering are bubble chains. Consider a graph $\mathcal{G}$ with $L$ loops in the nonrelativistic limit. In dimensional regularization, each loop will give a factor $M p / 4 \pi$, and there are $L+1$ vertices, each giving a factor $-i C_{2 m}^{\text {finite }} p^{2 m}$. If the operator $O_{2 m}$ appears $n_{m}$ times in the graph $\left(L+1=\Sigma_{m} n_{m}\right)$, the result is

$$
\mathcal{G}=\frac{4 \pi}{M} \prod_{m=0}^{\infty}\left(\frac{-i M C_{2 m}^{\text {finite }}}{4 \pi}\right)^{n_{m}} p^{j}
$$

where

$$
j=\sum_{m=0}^{\infty} 2 m n_{m}+L
$$

${ }^{3}$ For the scattering $N(\vec{q}+\vec{p})+N(\vec{q}-\vec{p}) \rightarrow N\left(\vec{q}+\vec{p}^{\prime}\right)+N\left(\vec{q}-\vec{p}^{\prime}\right)$ it is useful to define $p=\sqrt{M E_{\text {tot }}-\vec{q}^{2}+i \epsilon}$, where $E_{\text {tot }}$ is the total incoming energy, and $M$ is the nucleon mass. To simplify the notation we will work in the center-of-mass frame, $\vec{q}=0$, where $p^{2}$ $=\vec{p}^{2}=\vec{p}^{\prime 2}=M E$, and $E$ is the center-of-mass energy. For external particles, one can always translate between $E$ and $p$ using the equations of motion. 
If one matches onto the effective range expansion in the $\overline{\mathrm{MS}}$ scheme one finds $C_{2 m}^{\text {finite }} \sim 4 \pi a^{m+1} /\left(M \Lambda^{m}\right)$ [8]. We again see that all graphs $\mathcal{G}$ are proportional to $1 / M$, which is also true in PDS. The large $S$-wave scattering lengths enhance the importance of some graphs compared to the $p$ power counting. This affects the power counting for $S$-wave couplings and, through the mixing, couplings with $L$ and/or $L^{\prime}=2$ and $S=1$. For other channels we have the usual chiral power counting of $p$ 's. The power counting for insertions of fournucleon operators is [21]

$$
C_{2 m}^{(s)}\left(\mu_{R}\right) \mathcal{O}_{2 m}^{(s)} \sim C_{2 m}^{\left(L-L^{\prime}\right)}\left(\mu_{R}\right) p^{2 m} \sim Q^{q(s, m)},
$$

where

$$
\begin{aligned}
& q(s, m) \\
& = \begin{cases}m-1 & \text { for } L=L^{\prime}=0 \\
m & \text { for } S=1 \text { and }\left(L, L^{\prime}=0,2\right) \text { or }\left(L, L^{\prime}=2,0\right), \\
m+1 & \text { for } S=1 \text { and } L, L^{\prime}=2,2, \\
2 m & \text { for all other } S, L, \text { and } L^{\prime} .\end{cases}
\end{aligned}
$$

With the coefficients $C_{2 m}$ scaling as in Eq. (2.6), the graph $\mathcal{G}$ scales as

$$
\mathcal{G} \sim Q^{i}
$$

where

$$
i=\sum_{m} n_{m} q(s, m)+L
$$

Note that the power of $Q$ is less than or equal to the power of $p, i \leqslant j$. A useful mnemonic for this power counting is $1 / a$ $\sim Q$; however, the power counting is still valid for $Q a \gg 1$.

This $Q$ power counting will be manifest in any renormalization scheme in which the $C_{2 m}\left(\mu_{R}\right)$ scale with $\mu_{R} \sim Q$ in such a way that Eq. (2.6) is true. At leading order the counterterms $\delta^{n} C_{2 m}\left(\mu_{R}\right)$ will have the same $Q$ scaling as the coefficient $C_{2 m}\left(\mu_{R}\right)$. These schemes may differ by contributions in $C_{2 m}\left(\mu_{R}\right)$ that scale with a larger power of $\mu_{R} / \Lambda$, since this will not change the power counting at low momentum

Let us now discuss the theory with pions. To add pions, we identify them as the three pseudo-Goldstone bosons which arise from the breaking of chiral symmetry, $\mathrm{SU}(2)_{L}$ $\times \mathrm{SU}(2)_{R} \rightarrow \mathrm{SU}(2)_{V}$. With the pions included in this way, we are doing an expansion in $m_{\pi} / \Lambda_{\pi}$ and $p / \Lambda_{\pi}$. Note that in this theory, no matter how small $p$ is made the expansion parameter will never be smaller than $m_{\pi} / \Lambda_{\pi}$. This theory still includes the four-nucleon operators in Eq. (2.2), but the short distance physics parametrized by the coefficients $C_{2 m}$ is different because the pion is no longer integrated out. In the pion theory, the short distance $C_{2 m}$ coefficients should be independent of the scale $m_{\pi}$. All the $m_{\pi}$ dependence is now contained explicitly in powers of the light quark mass matrix in the Lagrangian.

Pions will be encoded in the representation, $\Sigma=\xi^{2}$ $=\exp (2 i \Pi / f)$, where

$$
\Pi=\left(\begin{array}{cc}
\pi^{0} / \sqrt{2} & \pi^{+} \\
\pi^{-} & -\pi^{0} / \sqrt{2}
\end{array}\right),
$$

and $f=130 \mathrm{MeV}$ is the pion decay constant. Under $\mathrm{SU}(2)_{L} \times \mathrm{SU}(2)_{R}$ the fields transform as $\Sigma \rightarrow L \Sigma R^{\dagger}, \quad \xi$ $\rightarrow L \xi U^{\dagger}=U \xi R^{\dagger}$, and $N \rightarrow U N$. The chiral covariant derivative is $D^{\mu}=\partial^{\mu}+\frac{1}{2}\left(\xi \partial^{\mu} \xi^{\dagger}+\xi^{\dagger} \partial^{\mu} \xi\right)$. With pions we have the following Lagrangian with terms involving zero, one, and two nucleons:

$$
\begin{aligned}
\mathcal{L}_{\pi}= & \frac{f^{2}}{8} \operatorname{Tr}\left(\partial^{\mu} \Sigma \partial_{\mu} \Sigma^{\dagger}\right)+\frac{f^{2} w}{4} \operatorname{Tr}\left(m_{q} \Sigma+m_{q} \Sigma^{\dagger}\right) \\
& +\frac{i g_{A}}{2} N^{\dagger} \sigma_{i}\left(\xi \partial_{i} \xi^{\dagger}-\xi^{\dagger} \partial_{i} \xi\right) N+N^{\dagger}\left(i D_{0}+\frac{\vec{D}^{2}}{2 M}\right) N \\
& -\sum_{s, m} C_{2 m}^{(s)} \mathcal{O}_{2 m}^{(s)}-D_{2}^{(s)} \omega \operatorname{Tr}\left(m^{\xi}\right)\left(N^{T} P_{i}^{(s)} N\right)^{\dagger}\left(N^{T} P_{i}^{(s)} N\right) \\
& +\cdots .
\end{aligned}
$$

Here $m^{\xi}=\frac{1}{2}\left(\xi m_{q} \xi+\xi^{\dagger} m_{q} \xi^{\dagger}\right), \quad m_{q}=\operatorname{diag}\left(m_{u}, m_{d}\right)$ is the quark mass matrix, $m_{\pi}^{2}=w\left(m_{u}+m_{d}\right)$ where $w$ is a constant, and $g_{A}=1.25$ is the nucleon axial-vector coupling. The ellipsis in Eq. (2.9) denotes terms with more derivatives and more powers of $m^{\xi}$.

With pions there are additional complications to the power counting $[12,23,13]$ which are similar to those encountered in nonrelativistic QED and QCD [9]. The complications arise because there are two relevant energy scales for the pions $E_{\pi} \sim Q^{2} / M$ for potential pions, and $E_{\pi} \sim Q$ for radiation pions. When the energy integral in loops is performed via contour integration, the graphs with potential pions come from terms in which one keeps the residue of a nucleon propagator pole. In these loops, the energy of the loop momentum is $\sim Q^{2} / M$ and the energy-dependent pieces of the pion propagator are suppressed by an additional $Q^{2} / M^{2}$. Nucleon propagators give a $Q^{-2}$ and the loop integrals give $Q^{5}$. There are also radiation pion graphs, in which the residue of the pion pole is kept. In these loops, the loop energy is $\sim Q$, the nucleon propagators give a $Q^{-1}$, and loop integrals give a $Q^{4}$. We find that adding a radiation pion to a bubble chain of contact interactions gives an additional suppression factor $Q^{N}$, where $N$ is the number of nucleon propagators in the radiation pion loop. In either case, a pion propagator gives a $Q^{-2}$, and each $\pi N N$ vertex gives a $Q$. The combined propagator and vertices for a single pion exchange give $Q^{0}$; so the pions can be treated perturbatively [3].

In general, pion exchange gives both long and short distance contributions. The short distance contributions from potential pions are important since they may limit the range of the effective field theory. A single potential pion exchange gives

$$
\begin{aligned}
& i \frac{g_{A}^{2}}{2 f^{2}} \frac{\vec{q} \cdot \vec{\sigma}_{\alpha \beta} \vec{q} \cdot \vec{\sigma}_{\gamma \delta}}{\vec{q}^{2}+m_{\pi}^{2}} \vec{\tau}_{1} \cdot \vec{\tau}_{2} \\
& \quad=i \frac{g_{A}^{2}}{2 f^{2}}\left[\frac{\vec{q} \cdot \vec{\sigma}_{\alpha \beta} \vec{q} \cdot \vec{\sigma}_{\gamma \delta}}{\vec{q}^{2}}-\frac{m_{\pi}^{2} \vec{q} \cdot \vec{\sigma}_{\alpha \beta} \vec{q} \cdot \vec{\sigma}_{\gamma \delta}}{\vec{q}^{2}\left(\vec{q}^{2}+m_{\pi}^{2}\right)}\right] \vec{\tau}_{1} \cdot \vec{\tau}_{2}
\end{aligned}
$$


where the spin indices connect to nucleon fields $N_{\alpha}^{\dagger} N_{\beta} N_{\gamma}^{\dagger} N_{\delta}$ which belong to external lines or propagators. The first term dominates for $\vec{q}^{2} \gg m_{\pi}^{2}$, and can be isolated by taking the limit $m_{\pi} \rightarrow 0$. Graphs with radiation pions are suppressed by $m_{\pi} / M$. In the nonrelativistic limit, with only potential pions, the only loop diagrams are ladders. Consider an arbitrary graph $\mathcal{G}$ with $n_{m}$ four point vertices $C_{2 m}^{\text {finite }}$ and $k$ potential pions. For $L$ loops, this graph has a total of $L+1=k$ $+\Sigma_{m} n_{m}$ vertices, and with $m_{\pi}=0$,

$$
\begin{aligned}
\mathcal{G} & \propto\left(\frac{M}{4 \pi}\right)^{L}\left(\frac{-i g_{A}^{2}}{2 f^{2}}\right)^{k} p^{j} \prod_{m=0}^{\infty}\left(-i C_{2 m}^{\text {finite }}\right)^{n_{m}} \\
& =\frac{4 \pi}{M}\left(\frac{-i M g_{A}^{2}}{8 \pi f^{2}}\right)^{k} p^{j} \prod_{m=0}^{\infty}\left(\frac{-i M C_{2 m}^{\text {finite }}}{4 \pi}\right)^{n_{m}},
\end{aligned}
$$

where

$$
j=\sum_{m=0}^{\infty} 2 m n_{m}+L
$$

In the ${ }^{1} S_{0}$ channel, the relation in Eq. (2.11) becomes an equality. The graph $\mathcal{G} \sim Q^{i}$ where $i$ is given in Eq. (2.7). The power counting of the $\delta^{\mathrm{uv}} C_{2 m}$ counterterms is determined by the need to cancel ultraviolet divergences, and will not spoil the scaling for the renormalized coefficients, since $i \leqslant j$. For graphs with only potential pions $\left(n_{m}=0\right)$, it appears that our expansion is in $p /(300 \mathrm{MeV})$ since

$$
\frac{M g_{A}^{2}}{8 \pi f^{2}} \simeq(300 \mathrm{MeV})^{-1}
$$

Comparing the size of potential pion graphs therefore predicts a range of $300 \mathrm{MeV}$, but the size of these graphs may change depending on the renormalization scheme (i.e., the finite subtractions). It is not known a priori how the contact interactions will affect the range of the effective theory. The scale $300 \mathrm{MeV}$ is therefore an approximate estimate for the range of the effective field theory with perturbative pions. A further discussion of this issue will be taken up in Sec. IV.

Next, consider the power counting for coefficients that multiply operators with powers of $m_{q}$. If we are interested in momenta of order $m_{\pi}$, then one counts $m_{q} \sim m_{\pi}^{2} \sim Q^{2}$. Therefore, any interaction term that has an operator with a total of $2 m$ powers of $p$ and $m_{\pi}$ will scale as $Q^{q(s, m)}$ where $q(s, m)$ is given in Eq. (2.6). For example, $D_{2}^{\left({ }^{1} S_{0}\right)} m_{\pi}^{2} \sim Q^{0}$. It is important to understand that in the KSW power counting $\mathrm{D}_{2}$ should be treated perturbatively even though the structure of the operator it multiplies is similar to that of the leading four-nucleon operator with no derivatives. Graphs with radiation pions will also give contributions with powers of $m_{\pi}^{2}$. In Ref. [1], the power counting for a general radiation pion graph is worked out; the only change is the power counting for the coefficients in Eq. (2.6).

\section{A. PDS scheme}

The PDS scheme is one scheme in which the KSW power counting is manifest. In the PDS scheme, we first let $d=4$ and take the $\delta^{\mathrm{uv}} C_{2 m}$ counterterms to subtract $1 / \epsilon$ poles as in the $\overline{\mathrm{MS}}$ scheme. We use the notation $\mu_{R}$ for the renormalization point and $\mu$ for the dimensional regularization parameter. In the PDS scheme, like in the $\overline{\mathrm{MS}}$ scheme, one takes $\mu=\mu_{R}$. In a momentum subtraction scheme this is not necessary. The next step in the PDS scheme is to take $d$ $=3$ and define the finite counterterms $\delta^{n} C_{2 m}\left(\mu_{R}\right)$ to subtract the $1 /(d-3)$ poles in the amplitude. Graphs which contribute are those whose vertices have a total of $2 m$ derivatives. When calculating the $\delta^{n} C_{2 m}\left(\mu_{R}\right)$ we take $m_{\pi}=0$ since, for instance, counterterms proportional to $m_{\pi}^{2}$ renormalize coefficients like $D_{2}\left(\mu_{R}\right)$. After making these subtractions everything is continued back to four dimensions. It is this second set of finite subtractions that gives the right power law dependence on $\mu_{R}$. To define the coefficients that multiply operators with powers of $m_{q}$, a similar procedure is followed except we count the powers of $m_{\pi}^{2}$ at the vertices. In the PDS scheme with just nucleons, all the graphs that affect the running of $C_{2 m}\left(\mu_{R}\right)$ are of order $Q^{q(s, m)}$, except for those with intermediate states of different orbital angular momenta. For example, the beta function for $C_{4}^{\left({ }^{3} S_{1}\right)}$ has contributions $\sim Q\left[q\left({ }^{3} S_{1}, 4\right)=1\right]$, as well as contributions $\sim Q^{3}$ from graphs with two $C_{2}^{\left({ }^{3} S_{1}-{ }^{3} D_{1}\right)}$ vertices. When pions are included there are additional graphs that are subleading in the power counting and effect the running of the couplings. In fact, in Sec. IV we will show that there will be corrections to the PDS beta function for $C_{0}^{\left({ }^{3} S_{1}\right)}\left(\mu_{R}\right)$ at all orders in $Q$.

\section{B. OS scheme}

Another renormalization scheme that can be used to reproduce the power counting in Eq. (2.6) is a momentum subtraction scheme. A simple physical definition for the renormalized couplings can be made by relating the couplings to the amplitude evaluated at the unphysical momentum $p=i \mu_{R}$. This scheme will be called the OS scheme, since in a relativistic field theory this would be referred to as an off-shell momentum subtraction scheme. We start by dividing up the full amplitude as

$$
i \mathcal{A}^{s}=i \sum_{m=0}^{\infty} \mathcal{A}_{2 m}^{s}+\cdots
$$

Here $\mathcal{A}_{2 m}^{s}$ contains the Feynman diagrams that will be used to define the coupling $C_{2 m}^{(s)}\left(\mu_{R}\right)$ (or equivalently the counterterms $\delta^{n} C_{2 m}$ ). The ellipsis in Eq. (2.13) denotes pieces that vanish as $m_{\pi} \rightarrow 0$ which are not needed to define $C_{2 m}\left(\mu_{R}\right)$. $A_{2 m}^{s}$ is defined to contain the remaining graphs that scale as $Q^{q(s, m)}$, where $q(s, m)$ is defined in Eq. (2.6). The definition for the renormalized coupling is then

$$
\left.i \mathcal{A}_{2 m}^{s}\right|_{\substack{p=i \mu_{R} \\ m_{\pi=0}}}=-i C_{2 m}^{(s)}\left(\mu_{R}\right)\left(i \mu_{R}\right)^{2 m} .
$$

As we will see, this ensures that $C_{2 m}\left(\mu_{R}\right)$ scales in the desired way. In general, there may be divergent graphs scaling as $Q^{i}$ and $p^{2 m}(i \leqslant 2 m)$ whose $1 / \epsilon$ poles need to be absorbed by a $\delta^{\mathrm{uv}} C_{2 m}$ counterterm. For example, consider the graph 


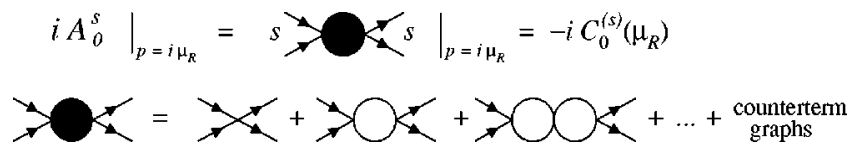

FIG. 2. Renormalization condition for $C_{0}\left(\mu_{R}\right)$. The amplitude $i \mathcal{A}^{s, 0}$ is the full four-point function with $C_{0}^{(s)}\left(\mu_{R}\right)$ and $\delta^{n} C_{0}^{(s)}\left(\mu_{R}\right)$ vertices, evaluated between incoming and outgoing states $s$ $={ }^{1} S_{0}$ or ${ }^{3} S_{1}$.

with two pions and one $C_{0}$ shown in row 4 of Fig. 4 , below. This graph has a $p^{2} / \epsilon$ pole which is canceled by a counterterm $\delta^{\mathrm{uv}} C_{2}$. The finite part of this graph is used in Eq. (2.14) to define $C_{4}\left(\mu_{R}\right)$ because the graph is order $Q$. The key point is that since $q(s, m) \leqslant 2 m$, an ultraviolet divergence that appears in a graph of a given order can always be absorbed into a coefficient that appeared at the same or lower order in the power counting. Therefore, we will define $\delta^{\mathrm{uv}} C_{2 m}$ in the $\overline{\mathrm{MS}}$ scheme to subtract all four-dimensional $1 / \epsilon$ poles so that these subtractions are independent of the renormalization point. The finite counterterms are then fixed by the renormalization condition in Eq. (2.14).

In the OS scheme, the coupling $C_{0}\left(\mu_{R}\right)$ is defined by the renormalization condition in Fig. 2 (with or without pions). This condition is to be imposed order by order in the loop expansion so that graphs with $n$ loops determine $\delta^{n} C_{0}\left(\mu_{R}\right)$. The $m_{\pi}=0$ part of pion graphs will also contribute to $C_{2 m}\left(\mu_{R}\right)$ for $m \geqslant 1$, in which case the condition $m_{\pi}=0$ in Eq. (2.14) is important. In the theory with pions, we also need to define couplings multiplying powers of $m_{q}$, like $D_{2}$ in Eq. (2.9). To define these couplings we will not include all the terms in the amplitude proportional to $m_{\pi}^{2}$. In particular, pion exchange graphs give long distance nonanalytic contributions which will not be used to define the running of the short distance coupling $D_{2}\left(\mu_{R}\right)$. The idea that long distance physics must be excluded from the short distance coefficients is discussed in Ref. [15]. A detailed discussion of how we define $D_{2}\left(\mu_{R}\right)$ in the OS scheme will be left to Sec. V.

Note that in the OS scheme there is another approach for calculating an amplitude in terms of renormalized couplings. One can calculate all loop graphs in $\mathcal{A}_{2 m}^{s}$ in terms of the finite (or $\overline{\mathrm{MS}}$ ) parameters and then demand that the renormalization condition in Eq. (2.14) is satisfied. This gives expressions for the renormalized couplings in terms of the constants $C_{2 m}^{\text {finite }}$. The amplitude can then be written in terms of renormalized couplings by inverting these equations. This simplifies higher order calculations.

In the OS scheme, when an amplitude is written in terms of renormalized couplings it will be explicitly $\mu_{R}$ independent at each order in $Q$. The $\mu_{R}$ dependence in the PDS scheme with pions is canceled by higher order terms. It is possible to obtain $\mu_{R}$ independent amplitudes in the PDS scheme if part of $C_{0}\left(\mu_{R}\right)$ is treated perturbatively [17]. The consequences of this $\mu_{R}$ dependence will be discussed in Sec. VI. In Sec. III, we will see that for the theory with just nucleons the OS scheme gives very similar definitions for the renormalized couplings to those in the PDS scheme. In Sec. $\mathrm{IV}$, we investigate the running couplings in both schemes in the theory with pions.

\section{THEORY WITH PIONS INTEGRATED OUT}

In this section, we compute the renormalized couplings in the nonrelativistic nucleon effective theory without pions.
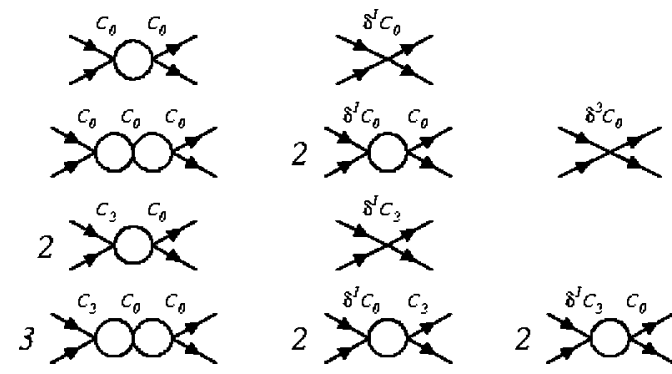

FIG. 3. One- and two-loop counterterms for $C_{0}$ and $C_{2}$. The solid lines are nucleon propagators, and symmetry factors are shown explicitly. The generalization to higher loops is straightforward.

We expect $\Lambda \sim m_{\pi}$. This theory will be examined in both PDS and OS schemes. The renormalization program is implemented by explicitly calculating the local counterterms. In Ref. [18], it is shown that the PDS and OS schemes give the same renormalized coupling constants in the ${ }^{1} S_{0}$ channel. Here we also consider the spin-triplet channel and higher derivative operators. Divergences in loop integrals are regulated using dimensional regularization. For the OS scheme, the same renormalization program can be carried out using a momentum cutoff regulator as shown in Appendix A. Following Ref. [3], we will multiply each loop integral by $(\mu / 2)^{(4-d)}$, and define $d=4-2 \epsilon$. Since there are no logarithmic divergences in the nucleon theory, $\delta^{\mathrm{uv}} C_{2 m}=0$ in dimensional regularization.

In both the PDS and OS schemes, it is straightforward to derive the finite counterterms $\delta^{n} C_{2 m}\left(\mu_{R}\right)$. The tree level graphs with $C_{0}\left(\mu_{R}\right)$ and $C_{2}\left(\mu_{R}\right)$ satisfy the renormalization condition in Eq. (2.14). Therefore, in both the PDS and OS schemes, $\delta^{0} C_{0}=\delta^{0} C_{2}=0$. At one and two loops we have the graphs in Fig. 3. In $d$ dimensions, the two graphs in the first row give

$$
\begin{aligned}
& \left(-i C_{0}\right)^{2}\left(\frac{-i M}{4 \pi}\right) \Gamma\left(\frac{3-d}{2}\right)\left(\frac{\mu}{2}\right)^{4-d}\left(\frac{-p^{2}-i \varepsilon}{4 \pi}\right)^{(d-3) / 2} \\
& +i \delta^{1} C_{0}
\end{aligned}
$$

determining $\delta^{1} C_{0}$. In the PDS scheme, we define the counterterm to cancel the $d=3$ pole in Eq. (3.1) and then continue back to four dimensions. In the OS scheme, we take $d=4$ and demand that the contribution to the amplitude in Eq. (3.1) satisfy the condition in Fig. 2. The counterterms calculated in each scheme are the same (with $\mu=\mu_{R}$ in the PDS scheme). In both schemes the counterterms determined from the graphs in Fig. 3 are

$$
\delta^{1} C_{0}\left(\mu_{R}\right)=\left(\frac{M \mu_{R}}{4 \pi}\right) C_{0}\left(\mu_{R}\right)^{2},
$$

$$
\delta^{2} C_{0}\left(\mu_{R}\right)=-\left(\frac{M \mu_{R}}{4 \pi}\right)^{2} C_{0}\left(\mu_{R}\right)^{3},
$$




$$
\begin{gathered}
\delta^{1} C_{2}\left(\mu_{R}\right)=2\left(\frac{M \mu_{R}}{4 \pi}\right) C_{2}\left(\mu_{R}\right) C_{0}\left(\mu_{R}\right), \\
\delta^{2} C_{2}\left(\mu_{R}\right)=-3\left(\frac{M \mu_{R}}{4 \pi}\right)^{2} C_{2}\left(\mu_{R}\right) C_{0}\left(\mu_{R}\right)^{2} .
\end{gathered}
$$

Note that it is essential that loop graphs also have vertices with insertions of the counterterms. For instance, the contribution to the amplitude from all the graphs in the second row of Fig. 3 is

$$
-i C_{0}\left(\mu_{R}\right)^{3}\left(\frac{M\left(i p+\mu_{R}\right)}{4 \pi}\right)^{2}
$$

If the one-loop graph with a $\delta^{1} C_{0}$ counterterm had been left out, then the answer would have been proportional to $\left(p^{2}\right.$ $+\mu_{R}^{2}$ ) which is not correct. Since the loops in the nucleon theory factorize, the renormalized $n$-loop graph gives (ip $\left.+\mu_{R}\right)^{n}$. Loop graphs will not always factorize once pions are included.

It is straightforward to extend this calculation to $n$ loops and to include higher derivatives. In both the OS and PDS schemes, this gives the following counterterms ( $s$ $\left.={ }^{1} S_{0},{ }^{3} S_{1}, n \geqslant 1\right)$ :

${ }^{1} S_{0}$ :

$$
\begin{aligned}
\delta^{n} C_{0}^{\left({ }^{1} S_{0}\right)}\left(\mu_{R}\right)= & (-1)^{n+1}\left(\frac{M \mu_{R}}{4 \pi}\right)^{n} C_{0}^{\left({ }^{1} S_{0}\right)}\left(\mu_{R}\right)^{n+1}, \\
\delta^{n} C_{2}^{\left({ }^{1} S_{0}\right)}\left(\mu_{R}\right)= & (-1)^{n+1}\left(\frac{M \mu_{R}}{4 \pi}\right)^{n}(n+1) \\
& \times C_{0}^{\left({ }^{1} S_{0}\right)}\left(\mu_{R}\right)^{n} C_{2}^{\left({ }^{1} S_{0}\right)}\left(\mu_{R}\right), \\
\delta^{n} C_{4}^{\left({ }^{1} S_{0}\right)}\left(\mu_{R}\right)= & (-1)^{n+1}\left(\frac{M \mu_{R}}{4 \pi}\right)^{n}(n+1) C_{0}^{\left({ }^{1} S_{0}\right)}\left(\mu_{R}\right)^{n-1} \\
& \times\left[C_{4}^{\left({ }^{1} S_{0}\right)}\left(\mu_{R}\right) C_{0}^{\left({ }^{1} S_{0}\right)}\left(\mu_{R}\right)+\frac{n}{2} C_{2}^{\left({ }^{1} S_{0}\right)}\left(\mu_{R}\right)^{2}\right] ;
\end{aligned}
$$

${ }^{3} S_{1},{ }^{3} D_{1}:$

$$
\begin{aligned}
\delta^{n} C_{0}^{\left({ }^{3} S_{1}\right)}\left(\mu_{R}\right)= & (-1)^{n+1}\left(\frac{M \mu_{R}}{4 \pi}\right)^{n} C_{0}^{\left({ }^{3} S_{1}\right)}\left(\mu_{R}\right)^{n+1}, \\
\delta^{n} C_{2}^{\left({ }^{3} S_{1}\right)}\left(\mu_{R}\right)= & (-1)^{n+1}\left(\frac{M \mu_{R}}{4 \pi}\right)^{n}(n+1) \\
& \times C_{0}^{\left({ }^{3} S_{1}\right)}\left(\mu_{R}\right)^{n} C_{2}^{\left({ }^{3} S_{1}\right)}\left(\mu_{R}\right),
\end{aligned}
$$

$$
\begin{aligned}
\delta^{n} C_{2}^{\left({ }^{3} S_{1}-{ }^{3} D_{1}\right)}\left(\mu_{R}\right)= & (-1)^{n+1}\left(\frac{M \mu_{R}}{4 \pi}\right)^{n} C_{0}^{\left({ }^{3} S_{1}\right)}\left(\mu_{R}\right)^{n} \\
& \times C_{2}^{\left({ }^{3} S_{1}-{ }^{3} D_{1}\right)}\left(\mu_{R}\right), \\
\delta^{n} C_{4}^{\left({ }^{3} D_{1}\right)}\left(\mu_{R}\right)= & (-1)^{n+1}\left(\frac{M \mu_{R}}{4 \pi}\right)^{n} C_{0}^{\left({ }^{3} S_{1}\right)}\left(\mu_{R}\right)^{n-1} \\
& \times\left[C_{2}^{\left({ }^{3} S_{1}-{ }^{3} D_{1}\right)}\left(\mu_{R}\right)\right]^{2} .
\end{aligned}
$$

Note that with $\mu_{R} \sim Q$, the counterterms have the same $Q$ scaling as their corresponding coupling constant. In the PDS scheme, there are also subleading terms that come from the mixing of angular momentum states. In the PDS scheme,

$$
\begin{aligned}
\delta^{n} C_{4}^{\left({ }^{3} S_{1}\right)}\left(\mu_{R}\right)= & (-1)^{n+1}\left(\frac{M \mu_{R}}{4 \pi}\right)^{n} C_{0}^{\left({ }^{3} S_{1}\right)}\left(\mu_{R}\right)^{n-1} \\
& \times\left[(n+1) C_{4}^{\left({ }^{3} S_{1}\right)}\left(\mu_{R}\right) C_{0}^{\left({ }^{3} S_{1}\right)}\left(\mu_{R}\right)\right. \\
& +\frac{n(n+1)}{2} C_{2}^{\left({ }^{3} S_{1}\right)}\left(\mu_{R}\right)^{2} \\
& \left.+n C_{2}^{\left({ }^{3} S_{1}-{ }^{3} D_{1}\right)}\left(\mu_{R}\right)^{2}\right]
\end{aligned}
$$

where the last term is suppressed by $Q^{2}$. In the OS scheme,

$$
\begin{aligned}
\delta^{n} C_{4}^{\left({ }^{3} S_{1}\right)}\left(\mu_{R}\right)= & (-1)^{n+1}\left(\frac{M \mu_{R}}{4 \pi}\right)^{n} C_{0}^{\left({ }^{3} S_{1}\right)}\left(\mu_{R}\right)^{n-1} \\
& \times\left[(n+1) C_{4}^{\left({ }^{3} S_{1}\right)}\left(\mu_{R}\right) C_{0}^{\left({ }^{3} S_{1}\right)}\left(\mu_{R}\right)\right. \\
& \left.+\frac{n(n+1)}{2} C_{2}^{\left({ }^{3} S_{1}\right)}\left(\mu_{R}\right)^{2}\right]
\end{aligned}
$$

which is the same as the ${ }^{1} S_{0}$ channel. In the OS scheme, graphs with two $C_{2}^{\left({ }^{3} S_{1}-{ }^{3} D_{1}\right)}$ couplings and any number of $C_{0}^{\left({ }^{3} S_{1}\right)}$, s contribute to the beta function for $C_{8}^{\left({ }^{3} S_{1}\right)}$ since they are order $Q^{3}$. One might also ask about channels where the large scattering length does not effect the power counting. In this case $C_{2 m}^{(s)}\left(\mu_{R}\right) \sim Q^{0}$, and we recover the usual chiral power counting. In our OS scheme, the counterterms $\delta^{n} C_{2 m}^{(s)}\left(\mu_{R}\right)$ in these channels are either zero or a constant independent of $\mu_{R}$.

From Eq. (2.4) one can derive the beta functions using

$$
\beta_{2 m} \equiv \mu_{R} \frac{\partial}{\partial \mu_{R}} C_{2 m}\left(\mu_{R}\right)=\sum_{n=0}^{\infty} \mu_{R} \frac{\partial}{\partial \mu_{R}} \delta^{n} C_{2 m}\left(\mu_{R}\right)
$$

The first few beta functions are 
${ }^{1} S_{0}:$

$$
\begin{aligned}
& \beta_{0}^{\left({ }^{1} S_{0}\right)}=\left(\frac{M \mu_{R}}{4 \pi}\right) C_{0}^{\left({ }^{1} S_{0}\right)}\left(\mu_{R}\right)^{2}, \\
& \left.\beta_{2}^{\left({ }^{1} S_{0}\right)}=2\left(\frac{M \mu_{R}}{4 \pi}\right) C_{0}^{\left({ }^{1} S_{0}\right)}\left(\mu_{R}\right) C_{2}^{(1} S_{0}\right)\left(\mu_{R}\right), \\
& \beta_{4}^{\left({ }^{1} S_{0}\right)}=\left(\frac{M \mu_{R}}{4 \pi}\right)\left[2 C_{4}^{\left({ }^{1} S_{0}\right)}\left(\mu_{R}\right) C_{0}^{\left({ }^{1} S_{0}\right)}\left(\mu_{R}\right)+C_{2}^{\left(1 S_{0}\right)}\left(\mu_{R}\right)^{2}\right] ;
\end{aligned}
$$

${ }^{3} S_{1},{ }^{3} D_{1}:$

$$
\begin{aligned}
\beta_{0}^{\left({ }^{3} S_{1}\right)} & =\left(\frac{M \mu_{R}}{4 \pi}\right) C_{0}^{\left({ }^{3} S_{1}\right)}\left(\mu_{R}\right)^{2}, \\
\beta_{2}^{\left({ }^{3} S_{1}\right)} & =2\left(\frac{M \mu_{R}}{4 \pi}\right) C_{0}^{\left({ }^{3} S_{1}\right)}\left(\mu_{R}\right) C_{2}^{\left({ }^{3} S_{1}\right)}\left(\mu_{R}\right), \\
\beta_{2}^{\left({ }^{3} S_{1}-{ }^{3} D_{1}\right)} & =\left(\frac{M \mu_{R}}{4 \pi}\right) C_{0}^{\left({ }^{3} S_{1}\right)}\left(\mu_{R}\right) C_{2}^{\left({ }^{3} S_{1}-{ }^{3} D_{1}\right)}\left(\mu_{R}\right),
\end{aligned}
$$

in agreement with Refs. [2,3]. For $S=0$ states the beta functions are one-loop exact in the sense that the contribution in Eq. (3.8) comes from the one-loop graphs, with the higher order graphs giving contributions which cancel. The reason for this cancellation is that the only loop corrections are in the bubble chain, and they form a geometric series. The sum of bubble graphs is just the chain of irreducible one-loop bubbles for the full (pointlike) propagator. An analogy would be QED, if the only possible graphs were the two-point photon graphs with electron loops. In this case the beta function would also be one-loop exact because the graphs that are not one-particle irreducible (1PI) do not contribute. In general, the beta functions of higher order couplings may have contributions beyond one loop in cases where angular momentum mixing is present.

Expressions for the running coupling constants can be derived by summing the counterterms in Eq. (2.4) or by solving renormalization group equations. For $s={ }^{1} S_{0}$ or ${ }^{3} S_{1}$ this gives

$$
\begin{aligned}
C_{0}^{(s)}\left(\mu_{R}\right) & =\frac{1}{1 / C_{0}^{\text {finite }}-M \mu_{R} /(4 \pi)}, \\
C_{2}^{(s)}\left(\mu_{R}\right) & =\frac{C_{2}^{\text {finite }}}{\left(C_{0}^{\text {finite }}\right)^{2}} \frac{1}{\left[1 / C_{0}^{\text {finite }}-M \mu_{R} /(4 \pi)\right]^{2}},
\end{aligned}
$$

where $C_{0}^{\text {finite }}$ and $C_{2}^{\text {finite }}$ are constants which can be determined by specifying boundary conditions. Since the theory should be good for arbitrarily small momenta, one possibility is to demand that the amplitude reproduce the effective range expansion, $p \cot (\delta)=-1 / a+\frac{1}{2} r_{0} p^{2}+\mathcal{O}\left(p^{4}\right)$. In Refs. [2,3] this matching was done at $\mu_{R}=0$, giving $C_{0}^{\text {finite }}=4 \pi a / M$, $C_{2}^{\text {finite }}=(4 \pi a / M) a r_{0} / 2$, etc. We could equally well have chosen a different matching point and obtained the same results. For $\mu_{R} \sim Q$, the running couplings in Eq. (3.9) have the scaling in Eq. (2.6). Written in terms of renormalized couplings the amplitude in the ${ }^{1} S_{0}$ or ${ }^{3} S_{1}$ channels is [3]

$$
\begin{aligned}
\mathcal{A}= & -\frac{4 \pi}{M}\left[\frac{1}{4 \pi /\left[M C_{0}\left(\mu_{R}\right)\right]+\mu_{R}+i p}\right. \\
& +\frac{4 \pi}{M} \frac{C_{2}\left(\mu_{R}\right)}{C_{0}\left(\mu_{R}\right)^{2}} \frac{p^{2}}{\left\{\left[4 \pi /\left[M C_{0}\left(\mu_{R}\right)\right]+\mu_{R}+i p\right]\right\}^{2}} \\
& +\mathcal{O}(Q)],
\end{aligned}
$$

and satisfies Eq. (2.14). The amplitude $\mathcal{A}$ is $\mu_{R}$ independent. It is interesting to note that we can choose a renormalization point where all loop corrections vanish, giving

$$
\begin{aligned}
\mathcal{A}^{s} & =\sum_{m=0}^{\infty} \mathcal{A}_{2 m}^{s}=-\sum_{m=1}^{\infty} C_{2 m}^{(s)}\left(\mu_{R}=-i p\right) p^{2 m} \\
& =-\frac{4 \pi}{M} \frac{1}{1 / a+i p}-\frac{4 \pi}{M}\left(\frac{1}{1 / a+i p}\right)^{2} \frac{r_{0}}{2} p^{2}+\cdots
\end{aligned}
$$

The amplitude exactly reproduces the effective range expansion by construction. From Eq. (3.11) the range of the effective field theory can be estimated as $\Lambda \sim 2 / r_{0} \sim m_{\pi}$ as expected.

It is possible to choose the boundary condition for $C_{0}\left(\mu_{R}\right)$ to change the location of the pole that appears at each order in the expansion. For processes involving the deuteron $[21,24]$ a more natural boundary condition is to choose the pole to appear at $-i p=\gamma=\sqrt{M E_{d}}$, and so $C_{0}^{\text {finite }}$ $=4 \pi /(M \gamma)$. To recover the effective range expansion, $C_{0}(\mu)$ is divided into nonperturbative and perturbative parts [17], $C_{0}\left(\mu_{R}\right)=C_{0}^{\mathrm{np}}\left(\mu_{R}\right)+C_{0}^{\mathrm{p}}\left(\mu_{R}\right)$, where $C_{0}^{\mathrm{np}}\left(\mu_{R}\right) \sim 1 / Q$ and $C_{0}^{\mathrm{p}}\left(\mu_{R}\right) \sim Q^{0}$. In this case the amplitude becomes

$$
\begin{aligned}
\mathcal{A}^{s}= & -\frac{4 \pi}{M}\left[\frac{1}{\gamma+i p}+\frac{4 \pi}{M} \frac{C_{0}^{\mathrm{p}}\left(\mu_{R}\right)}{\left[C_{0}^{\mathrm{np}}\left(\mu_{R}\right)\right]^{2}} \frac{1}{(\gamma+i p)^{2}}\right. \\
& \left.+\frac{4 \pi}{M} \frac{C_{2}\left(\mu_{R}\right)}{\left[C_{0}^{\mathrm{np}}\left(\mu_{R}\right)\right]^{2}} \frac{1}{(\gamma+i p)^{2}} p^{2}\right],
\end{aligned}
$$

where the first term is of order $1 / Q$, and the second and third terms are of order $Q^{0}$. The renormalization group equations (RGE's) are

$$
\begin{gathered}
\mu_{R} \frac{\partial}{\partial \mu_{R}} C_{0}^{\mathrm{np}}\left(\mu_{R}\right)=\frac{M \mu_{R}}{4 \pi} C_{0}^{\mathrm{np}}\left(\mu_{R}\right)^{2}, \\
\mu_{R} \frac{\partial}{\partial \mu_{R}} C_{0}^{\mathrm{p}}\left(\mu_{R}\right)=2 \frac{M \mu_{R}}{4 \pi} C_{0}^{\mathrm{np}}\left(\mu_{R}\right) C_{0}^{\mathrm{p}}\left(\mu_{R}\right)+\mathcal{O}(Q) .
\end{gathered}
$$

These can be derived by substituting $C_{0}\left(\mu_{R}\right)=C_{0}^{\mathrm{np}}\left(\mu_{R}\right)$ $+C_{0}^{\mathrm{p}}\left(\mu_{R}\right)$ into the renormalization group equation for $C_{0}\left(\mu_{R}\right)$. They can also be derived using the counterterm method described above. If we demand that the observed scattering length and effective range be reproduced at this order, then we find 


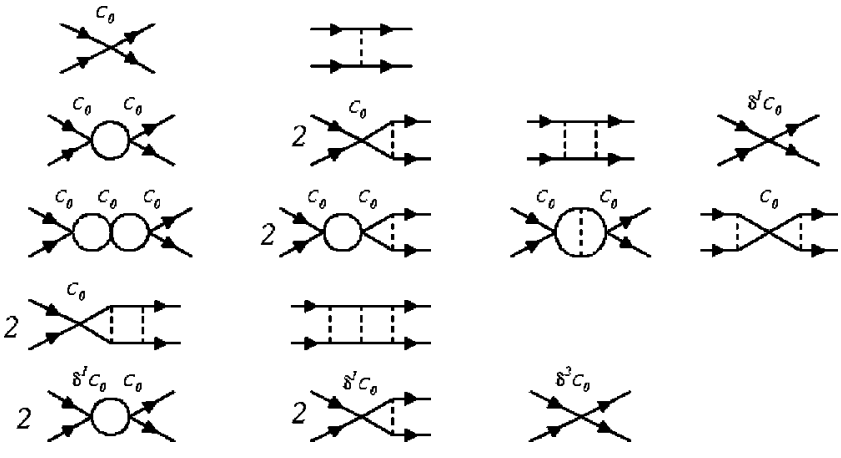

FIG. 4. Zero-, one-, and two-loop graphs with $C_{0}$ and $\delta^{n} C_{0}$ vertices and potential pion exchange. The dashed lines denote potential pion propagators.

$$
\frac{4 \pi}{M} \frac{C_{0}^{\mathrm{p}}\left(\mu_{R}\right)}{\left[C_{0}^{\mathrm{np}}\left(\mu_{R}\right)\right]^{2}}=\gamma-\frac{1}{a}, \quad \frac{4 \pi}{M} \frac{C_{2}\left(\mu_{R}\right)}{\left[C_{0}^{\mathrm{np}}\left(\mu_{R}\right)\right]^{2}}=\frac{r_{0}}{2} .
$$

In order for the power counting of $C_{0}^{\mathrm{p}}\left(\mu_{R}\right)$ to be consistent, we must treat $\gamma-1 / a \sim Q^{2}$.

\section{THEORY WITH NUCLEONS AND PIONS}

In this section, we study the renormalization of contact interactions in the effective field theory with pions. In the ${ }^{3} S_{1}$ channel, graphs with two or more consecutive potential pions do not factorize and give poles of the form $p^{n} / \epsilon$. We explicitly compute these poles for two-loop pion graphs. There are also $m_{\pi}^{2} / \epsilon$ poles in both the ${ }^{1} S_{0}$ and ${ }^{3} S_{1}$ channels at order $Q^{0}[2,3]$. Because of these $1 / \epsilon$ poles, pions cannot be summed to all orders in a model-independent way. The finite counterterms in the PDS and OS schemes are different in this theory. Throughout this section we will take $m_{\pi}=0$, since we are only interested in the couplings $C_{2 m}\left(\mu_{R}\right)$. The $D_{2}\left(\mu_{R}\right)$ counterterms will be considered in Sec. V. We compute the PDS counterterms and beta functions for $C_{0}\left(\mu_{R}\right)$ and $C_{2}\left(\mu_{R}\right)$ to order $Q$. In the PDS scheme, $C_{0}\left(\mu_{R}\right)$ no longer obeys the $Q$ scaling for $\mu_{R} \gtrsim 300 \mathrm{MeV}$ [3]. This can be fixed by treating part of the coupling $C_{0}\left(\mu_{R}\right)$ perturbatively as discussed in Sec. VI. The exact expressions for $C_{0}\left(\mu_{R}\right), C_{2}\left(\mu_{R}\right)$, and $C_{4}\left(\mu_{R}\right)$ are given in the OS scheme and exhibit the correct $Q$ scaling for all $\mu_{R}>1 / a$. Therefore, it is no longer apparent that the power counting breaks down at $300 \mathrm{MeV}$. The $300 \mathrm{MeV}$ scale does appear in the short distance contribution to the amplitude from pion exchange; however, it can only be taken as an estimate for the range of the effective field theory once pion and contact interactions are both included. In Sec. VI, we will discuss how experimental data suggest that $\Lambda_{\pi} \gtrsim 300 \mathrm{MeV}$.

To determine how the pions contribute to the beta functions for $C_{2 m}\left(\mu_{R}\right)$, we use the rules in Sec. II. Some of the pion graphs that will be needed are shown in Fig. 4.

In both the PDS and OS schemes, the first step is to subtract $1 / \epsilon$ poles. For two nucleons in the ${ }^{1} S_{0}$ channel the spinor indices in Eq. (2.10) are contracted with $\delta_{\alpha \delta} \delta_{\beta \gamma}$. Therefore the $m_{\pi}=0$ piece of pion exchange reduces to a contact interaction and gives no $1 / \epsilon$ poles. In the ${ }^{3} S_{1}$ channel, graphs with two or more consecutive pions do not factorize and may have $1 / \epsilon$ poles. Order $Q$ graphs with two consecutive potential pions are shown in the first row of Fig. 5 , and labeled $(a),(b)$, and $(c)$. We find

$$
\begin{aligned}
(a)= & -i \frac{3}{2}\left(\frac{g_{A}^{2}}{2 f^{2}}\right)^{2}\left(\frac{-i p M}{4 \pi}\right), \\
(b)= & -3 i C_{0}^{\text {finite }}\left(\frac{g_{A}^{2}}{2 f^{2}}\right)^{2}\left(\frac{-i p M}{4 \pi}\right)^{2} \\
& \times\left[\frac{1}{\epsilon}-2 \gamma+\frac{14}{3}-4 \ln (2)+2 \ln \left(\frac{\pi \mu^{2}}{-p^{2}-i \varepsilon}\right)\right], \\
(c)= & -3 i\left(C_{0}^{\text {finite }}\right)^{2}\left(\frac{g_{A}^{2}}{2 f^{2}}\right)^{2}\left(\frac{-i p M}{4 \pi}\right)^{3} \\
& \times\left[\frac{1}{\epsilon}-3 \gamma-6 \ln 2+\frac{37}{6}+3 \ln \left(\frac{\pi \mu^{2}}{-p^{2}-i \varepsilon}\right)\right] .
\end{aligned}
$$

Graphs $(b)$ and $(c)$ have been written with $C_{0}^{\text {finite }}$ vertices to emphasize that the uv counterterm which cancels their divergent part is independent of $\mu_{R}$. The divergence in $(b)$ is canceled by a tree level graph with the counterterm

$$
\delta^{2, \mathrm{uv}} C_{2}=-6 C_{0}^{\text {finite }}\left(\frac{M g_{A}^{2}}{8 \pi f^{2}}\right)^{2}\left[\frac{1}{2 \epsilon}-\gamma+\ln (\pi)+2-2 \ln (2)\right] \text {, }
$$

where the superscript 2 indicates that the counterterm comes in at two loops. The extra factor $2-2 \ln (2)$ is included because this leads to simpler analytic expressions. Expanding the $C_{0}$ bubble graph (second row, first column of Fig. 4) in $\epsilon$ gives

$$
-\frac{p M}{4 \pi}\left(C_{0}\right)^{2}\left\{1+\epsilon\left[2-\gamma-2 \ln (2)+\ln \left(\frac{\pi \mu^{2}}{-p^{2}-i \epsilon}\right)\right]\right\} .
$$

When graphs with $1 / \epsilon$ poles are dressed with $C_{0}$ bubbles, the factors of $[2-\gamma-2 \ln 2+\ln (\pi)]$ that appear are canceled by similar factors from the counterterms. In fact, $\delta^{2 \text {,uv }} C_{2}$ is the only uv counterterm we need for two-potential-pion exchange with $m_{\pi}=0$. The $1 / \epsilon$ pole in $(c)$ is nonanalytic since it is proportional to $p^{3}$. When graph $(c)$ is added to graphs $(d)$ and $(e)$ the poles cancel. These cancellations continue to occur when more $C_{0}$ bubbles are added to $(b)$ and $(c)$. After including graphs with $\delta^{2, \text { uv }} C_{2}$ we find

$$
\begin{aligned}
(b)+i \delta^{2, \mathrm{uv}} C_{2} p^{2}= & -3 i C_{0}^{\text {finite }}\left(\frac{g_{A}^{2}}{2 f^{2}}\right)^{2}\left(\frac{-i p M}{4 \pi}\right)^{2} \\
& \times\left[\frac{2}{3}+2 \ln \left(\frac{\mu^{2}}{-p^{2}-i \varepsilon}\right)\right],
\end{aligned}
$$




$$
\begin{aligned}
(c)+\frac{1}{2}(e)= & -3 i\left(C_{0}^{\text {finite }}\right)^{2}\left(\frac{g_{A}^{2}}{2 f^{2}}\right)^{2}\left(\frac{-i p M}{4 \pi}\right)^{3} \\
& \times\left[\frac{1}{6}+2 \ln \left(\frac{\mu^{2}}{-p^{2}-i \varepsilon}\right)\right] .
\end{aligned}
$$

Note that for $\mu \sim p$ there are no large numerical factors from these graphs.

In the ${ }^{3} S_{1}$ channel, potential pion graphs without contact interactions also have $p^{2} / \epsilon$ poles. The two-loop graph with three potential pions (fourth row, second column in Fig. 4) is equal to

$$
\frac{4 \pi i}{M}\left(\frac{M g_{A}^{2}}{8 \pi f^{2}}\right)^{3} p^{2}\left[\frac{3}{\epsilon}+\cdots\right] .
$$

In the $Q$ power counting, this graph is order $Q^{2}$ and will not be considered here. Because of these $1 / \epsilon$ poles, it is not possible to sum pion ladder graphs to all orders. Now that the ultraviolet divergences have been removed from graphs $(b)$ and $(c)$, the finite subtractions can be performed.

\section{A. PDS scheme}

For the PDS scheme in the ${ }^{1} S_{0}$ channel, we can compute the effect of potential pions on the $C_{2 m}\left(\mu_{R}\right)$ counterterms to all orders in $Q$ (neglecting relativistic corrections). For $C_{0}\left(\mu_{R}\right)$, the relevant zero-, one-, and two-loop graphs are shown in Fig. 4. The $C_{0}\left(\mu_{R}\right)$ and $C_{2}\left(\mu_{R}\right)$ counterterms are

$$
\begin{aligned}
\delta^{n} C_{0}^{\left({ }^{1} S_{0}\right)}\left(\mu_{R}\right)= & (-1)^{n+1}\left(\frac{M \mu_{R}}{4 \pi}\right)^{n}\left[C_{0}\left(\mu_{R}\right)+\frac{g_{A}^{2}}{2 f^{2}}\right]^{n+1}, \\
\delta^{n} C_{2}^{\left({ }^{1} S_{0}\right)}\left(\mu_{R}\right)= & (-1)^{n+1}(n+1)\left(\frac{M \mu_{R}}{4 \pi}\right)^{n} \\
& \times\left[C_{0}\left(\mu_{R}\right)+\frac{g_{A}^{2}}{2 f^{2}}\right]^{n} C_{2}\left(\mu_{R}\right) .
\end{aligned}
$$

The PDS counterterms in the ${ }^{3} S_{1}$ channel will only be computed to order $Q$ since the loop graphs with consecutive pions do not factorize. For this case it is essential to use the counterterms to carry out the PDS renormalization program. To define $C_{0}\left(\mu_{R}\right)$ at order $Q$, we set up the finite subtractions as in Fig. 4, but leave out all graphs with more than two potential pions since they are $\mathcal{O}\left(Q^{2}\right)$ (we also neglect relativistic corrections that are order $Q$ but come with an additional $\left.1 / M^{2}\right)$. Note that in $d=3$ only the overall divergence $\left[\propto 1 /(d-3)^{n}\right.$ for $n$ loops] is needed since loops with counterterms will cancel the sub-divergences. Evaluating the graphs in Fig. 5 with $d=3$ and then continuing back to $d$ $=4$ gives

$$
\begin{aligned}
& (a)=-9 i\left(\frac{g_{A}^{2}}{2 f^{2}}\right)^{2}\left(\frac{\mu_{R} M}{4 \pi}\right), \\
& (b)=-12 i C_{0}\left(\mu_{R}\right)\left(\frac{g_{A}^{2}}{2 f^{2}}\right)^{2}\left(\frac{\mu_{R} M}{4 \pi}\right)^{2},
\end{aligned}
$$

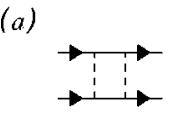

(b)

(d)

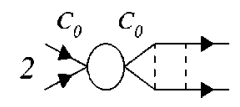

(c)

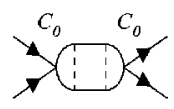

FIG. 5. The basic order $Q$ graphs in the ${ }^{3} S_{1}$ channel whose loop integrals do not factorize even for $m_{\pi}=0$.

$$
(c)=-5 i C_{0}\left(\mu_{R}\right)^{2}\left(\frac{g_{A}^{2}}{2 f^{2}}\right)^{2}\left(\frac{\mu_{R} M}{4 \pi}\right)^{3} \text {. }
$$

Using these values we find

$$
\begin{aligned}
\delta^{1} C_{0}^{\left({ }^{3} S_{1}\right)}= & \left(\frac{M \mu_{R}}{4 \pi}\right)\left[C_{0}\left(\mu_{R}\right)^{2}+2 C_{0}\left(\mu_{R}\right) \frac{g_{A}^{2}}{2 f^{2}}+9\left(\frac{g_{A}^{2}}{2 f^{2}}\right)^{2}\right], \\
\delta^{n} C_{0}^{\left({ }^{3} S_{1}\right)}= & (-1)^{n+1}\left(\frac{M \mu_{R}}{4 \pi}\right)^{n}\left[C_{0}\left(\mu_{R}\right)^{n+1}\right. \\
& +(n+1) C_{0}\left(\mu_{R}\right)^{n} \frac{g_{A}^{2}}{2 f^{2}}+\frac{1}{2}(n+1) \\
& \left.\times(n+4) C_{0}\left(\mu_{R}\right)^{n-1}\left(\frac{g_{A}^{2}}{2 f^{2}}\right)^{2}\right] \text { for } n \geqslant 2 . \quad 4.8
\end{aligned}
$$

Note that for graphs with two consecutive potential pions, the $\mu_{R}$ dependence does not come in the linear combination $\mu_{R}+i p$. For instance, adding the PDS counterterm to graph (a) in Fig. 5 gives the linear combination $3 \mathrm{ip} / 2+9 \mu_{R}$.

In the PDS scheme, like in the $\overline{\mathrm{MS}}$ scheme, the renormalized coupling $C_{2}\left(\mu_{R}\right)$ will depend on $\ln \left(\mu_{R}^{2} / \mu_{0}^{2}\right)$ in such a way that the $\ln \left(\mu_{R}^{2}\right)$ dependence in the amplitudes in Eq. (4.1) is canceled. Here $\mu_{0}$ is an arbitrary scale expected to be of $\operatorname{order} \Lambda_{\pi}$. At order $Q$ we find

$$
\begin{aligned}
\delta^{1} C_{2}^{\left({ }^{3} S_{1}\right)}\left(\mu_{R}\right)= & 2\left(\frac{M \mu_{R}}{4 \pi}\right)\left[C_{0}\left(\mu_{R}\right)+\frac{g_{A}^{2}}{2 f^{2}}\right] C_{2}\left(\mu_{R}\right), \\
\delta^{n} C_{2}^{\left({ }^{3} S_{1}\right)}\left(\mu_{R}\right)= & (-1)^{n+1}\left\{(n+1)\left(\frac{M \mu_{R}}{4 \pi}\right)^{n}\right. \\
& \times\left[C_{0}\left(\mu_{R}\right)^{n}+n \frac{g_{A}^{2}}{2 f^{2}} C_{0}\left(\mu_{R}\right)^{n-1}\right] C_{2}\left(\mu_{R}\right) \\
& +6\left(\frac{M \mu_{R}}{4 \pi}\right)^{n-2} \quad C_{0}\left(\mu_{R}\right)^{n-1}\left(\frac{M g_{A}^{2}}{8 \pi f^{2}}\right)^{2} \\
& \left.\times \ln \left(\frac{\mu_{R}^{2}}{\mu_{0}^{2}}\right)\right\} \quad \text { for } n \geqslant 2 .
\end{aligned}
$$

Note that the part of $\delta^{n} C_{2}\left(\mu_{R}\right)$ proportional to $\ln \left(\mu_{R}^{2} / \mu_{0}^{2}\right)$ has a coefficient that sums up to $C_{0}^{\text {finite }}$ at this order. From Eqs. (4.6), (4.8), and (4.9) we find 


$$
\begin{aligned}
\beta_{0}^{\left({ }^{1} S_{0}\right)=} & \frac{M \mu_{R}}{4 \pi}\left[C_{0}\left(\mu_{R}\right)+\frac{g_{A}^{2}}{2 f^{2}}\right]^{2}, \\
\beta_{2}^{\left({ }^{1} S_{0}\right)=}= & \frac{M \mu_{R}}{4 \pi}\left[C_{0}\left(\mu_{R}\right)+\frac{g_{A}^{2}}{2 f^{2}}\right] C_{2}\left(\mu_{R}\right), \\
\beta_{0}^{\left({ }^{3} S_{1}\right)}= & \frac{M \mu_{R}}{4 \pi}\left\{C_{0}^{2}+2 \frac{g_{A}^{2}}{2 f^{2}} C_{0}+9+4\left(\frac{\mu_{R} M C_{0}}{4 \pi}\right)\right. \\
& \left.\left.+2\left(\frac{\mu_{R} M C_{0}}{4 \pi}\right)^{2}\right]\left(\frac{g_{A}^{2}}{2 f^{2}}\right)^{2}\right\}+\mathcal{O}\left(Q^{2}\right), \\
\beta_{2}^{\left({ }^{3} S_{1}\right)}= & 2 \frac{M \mu_{R}}{4 \pi}\left[C_{0}\left(\mu_{R}\right)+\frac{g_{A}^{2}}{2 f^{2}}\right] C_{2}\left(\mu_{R}\right)-12\left(\frac{M g_{A}^{2}}{8 \pi f^{2}}\right)^{2} \\
& \times C_{0}\left(\mu_{R}\right)\left[1+\mu_{R} \frac{M}{4 \pi} C_{0}\left(\mu_{R}\right)\right]+\mathcal{O}\left(Q^{0}\right) .(4.10)
\end{aligned}
$$

Note that in the ${ }^{1} S_{0}$ channel all contributions to the beta functions beyond one loop cancel, leaving them one-loop exact. In Ref. [3], the last two terms in $\beta_{0}^{\left({ }^{3} S_{1}\right)}$ are absent, but should be included in the complete order $Q$ calculation. Dimensional analysis implies that the ${ }^{3} S_{1}$ beta functions can have corrections at all higher orders in $Q$, since there is nothing to prevent the dimensionless factor $\left(\mu_{R} g_{A}^{2} M\right) /\left(8 \pi f^{2}\right)$ $\sim Q$ from appearing. In Ref. [15], expressions for the beta functions are derived by demanding that $\partial \mathcal{A} / \partial \mu_{R}=0$, but these are not the PDS beta functions. Since in all renormalization schemes $\partial \mathcal{A} / \partial \mu_{R}=0$, this condition is not sufficient to fix the renormalization scheme uniquely. As discussed in Ref. [17], the large $\mu_{R}$ behavior of $C_{0}^{\left({ }^{3} S_{1}\right)}\left(\mu_{R}\right)$ is unknown because of the higher order corrections.

\section{B. OS scheme}

In the OS scheme, there is no such ambiguity since at a given order in $Q$ the running of all the coupling constants that enter at that order is known exactly. The coupling $C_{0}^{(s)}\left(\mu_{R}\right)$ has contributions only from the nucleon graphs discussed in Sec. II and therefore has the same beta function. For $C_{2}^{(s)}\left(\mu_{R}\right)$, the order $Q^{0}$ graphs in $\mathcal{A}_{2}$ include the nucleon graphs from Sec. II, as well as the graphs with one potential pion and any number of $C_{0}$ vertices. At the tree level we add a finite counterterm to cancel the $m_{\pi}=0$ part of the tree level pion interaction at $p=i \mu_{R}$ :

$$
\delta^{0} C_{2}^{(s)}\left(\mu_{R}\right)=-\frac{g_{A}^{2}}{2 f^{2}} \frac{1}{\mu_{R}^{2}} .
$$

This counterterm is of order $Q^{-2}$ like $C_{2}\left(\mu_{R}\right)$ itself. Since all the graphs in $\mathcal{A}_{2}$ factorize, the higher loop counterterms are the same as in the theory without pions; so $\delta^{n} C_{2}$ for $n \geqslant 1$ are given in Eq. (3.4). The exact beta function is then

$$
\begin{aligned}
\beta_{2}^{(s)}= & 2 \frac{M \mu_{R}}{4 \pi} C_{0}\left(\mu_{R}\right) C_{2}\left(\mu_{R}\right) \\
& +2 \frac{g_{A}^{2}}{2 f^{2}}\left(1+\frac{M \mu_{R}}{4 \pi} C_{0}\left(\mu_{R}\right)\right)^{2} \frac{1}{\mu_{R}^{2}} .
\end{aligned}
$$

Note that the finite $\ln \left[\mu^{2} /\left(-p^{2}-i \epsilon\right)\right]$ terms in Eq. (4.1) are order $Q$ and in the OS scheme do not affect the running of $C_{2}\left(\mu_{R}\right)$, but rather $C_{4}\left(\mu_{R}\right)$. In terms of the finite constants $C_{0}^{\text {finite }}$ and $C_{2}^{\text {finite }}$ we have the solutions

$$
\begin{gathered}
C_{0}^{(s)}\left(\mu_{R}\right)=\frac{1}{1 / C_{0}^{\text {finite }}-M \mu_{R} /(4 \pi)}, \\
C_{2}^{(s)}\left(\mu_{R}\right)=\frac{C_{2}^{\text {finite }}-g_{A}^{2} /\left(2 f^{2} \mu_{R}^{2}\right)}{\left[1-\mu_{R} C_{0}^{\text {finite }} M /(4 \pi)\right]^{2}} .
\end{gathered}
$$

Although it may seem that the piece of $C_{2}^{(s)}\left(\mu_{R}\right)$ that goes as $1 / \mu_{R}^{4}$ will spoil the power counting for low momentum, in fact, the $1 / \mu_{R}^{2}$ part dominates entirely until $\mu_{R} \sim 1 / a$, since $C_{0}^{\text {finite }} \sim a, C_{2}^{\text {finite }} \sim a^{2}$. Written in terms of renormalized couplings the $m_{\pi}=0$ part of the next-to-leading order OS amplitude is

$$
\begin{aligned}
& \frac{-C_{2}\left(\mu_{R}\right) p^{2}}{\left[1+\left(\mu_{R}+i p\right) M C_{0}\left(\mu_{R}\right) /(4 \pi)\right]^{2}} \\
& -\frac{g_{A}^{2}}{2 f^{2}} \frac{\mu_{R}^{2}+p^{2}}{\mu_{R}^{2}} \frac{\left[1+\mu_{R} C_{0}\left(\mu_{R}\right) M /(4 \pi)\right]^{2}}{\left[1+\left(\mu_{R}+i p\right) C_{0}\left(\mu_{R}\right) M /(4 \pi)\right]^{2}},
\end{aligned}
$$

which is of order $Q^{0}$ as desired.

One might still ask if the problem with the $300 \mathrm{MeV}$ scale will reappear in higher order coefficients. To check that this is not the case we compute the running of the coupling $C_{4}\left(\mu_{R}\right)$ in the OS scheme. The easiest way to compute this running coupling constant is to compute the order $Q$ amplitude in terms of the finite couplings $C_{2 m}^{\text {finite }}$ and then demand that the amplitude satisfy the renormalization condition in Eq. (2.14). The graphs we need to compute include those with

(i) one $C_{4}$ and any number of $C_{0}$ 's,

(ii) two $C_{2}$ 's and any number of $C_{0}$ 's,

(iii) one $C_{2}$, one potential pion, and any number of $C_{0}$ 's,

(iv) two potential pions and any number of $C_{0}$ 's.

Computing these graphs in terms of the finite couplings and then demanding that the amplitudes satisfy the renormalization condition gives the OS couplings 


$$
\begin{aligned}
C_{4}^{\left({ }^{1} s_{0}\right)}\left(\mu_{R}\right)= & \frac{C_{4}^{\text {finite }}}{\left[1-\mu_{R} M /(4 \pi) C_{0}^{\text {finite }}\right]^{2}} \\
& +\frac{\mu_{R} M}{4 \pi} \frac{\left[C_{2}^{\text {finite }}-g_{A}^{2} /\left(2 f^{2} \mu_{R}^{2}\right)\right]^{2}}{\left[1-\mu_{R} M /(4 \pi) C_{0}^{\text {finite }}\right]^{3}}, \\
C_{4}^{\left({ }^{3} S_{1}\right)}\left(\mu_{R}\right)= & \frac{C_{4}^{\text {finite }}}{\left[1-\mu_{R} M /(4 \pi) C_{0}^{\text {finite }}\right]^{2}} \\
& +\frac{\mu_{R} M}{4 \pi} \frac{\left[C_{2}^{\text {finite }}-g_{A}^{2} /\left(2 f^{2}\right) / 1 / \mu_{R}^{2}\right]^{2}}{\left[1-\mu_{R} M /(4 \pi) C_{0}^{\text {finite }}\right]^{3}} \\
& +\frac{1}{2}\left(\frac{g_{A}^{2}}{2 f^{2}}\right)^{2} \frac{M}{4 \pi} \frac{1}{\mu_{R}^{3}} \frac{\left[1-2 \mu_{R} M /(4 \pi) C_{0}^{\text {finite }}\right]}{\left[1-\mu_{R} M /(4 \pi) C_{0}^{\text {finite }}\right]^{2}} \\
& -6\left(\frac{M g_{A}^{2}}{8 \pi f^{2}}\right)^{2} \frac{C_{0}^{\text {finite }} \ln \left(\mu_{R}^{2} / \mu^{2}\right)}{\mu_{R}^{2}\left[1-\mu_{R} M /(4 \pi) C_{0}^{\text {finite }}\right]},
\end{aligned}
$$

where here $\mu$ is an unknown scale expected to be of order $\Lambda_{\pi}$. Again the pion contributions do not spoil the $\mu_{R}$ scaling behavior, since they are suppressed by factors of the large scattering length. Note that at order $Q$ the PDS coupling $C_{4}\left(\mu_{R}\right)$ [3] is the $g_{A} \rightarrow 0$ limit of Eq. (4.16).

In this section, expressions for the renormalized couplings $C_{0}\left(\mu_{R}\right), C_{2}\left(\mu_{R}\right)$, and $C_{4}\left(\mu_{R}\right)$ were derived in the PDS and OS schemes working to order $Q$. For the ${ }^{3} S_{1}$ channel, we have shown that $C_{0}\left(\mu_{R}\right)$ has corrections at all orders in $Q$ in the PDS scheme. Unlike the PDS scheme, the OS couplings $C_{2 m}\left(\mu_{R}\right)$ can be computed exactly because they only have contributions at one order in $Q$. The OS couplings exhibit the correct $\mu_{R}$ scaling for all $\mu_{R}>1 / a$.

\section{COUPLING $D_{2}\left(\mu_{R}\right)$}

In this section, the OS and PDS counterterms for $D_{2}\left(\mu_{R}\right)$ are computed. To define $D_{2}\left(\mu_{R}\right)$ in the OS scheme, we take

$$
\left.i A^{s}\left(D_{2}\right)\right|_{p=i \mu_{R}}=-i D_{2}^{(s)}\left(\mu_{R}\right) m_{\pi}^{2},
$$

where $\mathcal{A}^{s}\left(D_{2}\right)$ contains terms in the amplitude that are analytic in $m_{\pi}^{2}$ and proportional to $m_{\pi}^{2}$. Only terms that are analytic in $m_{\pi}^{2}$ are kept because it is unnatural to put long distance nonanalytic contributions that come from pion exchange into the definition of the short distance coupling [15]. For example, one potential pion exchange gives a $m_{\pi}^{2} / p^{2} \ln \left(1+4 p^{2} / m_{\pi}^{2}\right)$ term. Including this in $A^{s}\left(D_{2}\right)$ would give $D_{2}\left(\mu_{R}\right)$ both a branch cut at $\mu_{R}=m_{\pi} / 2$ as well as an explicit dependence on the scale $m_{\pi}$. In the OS scheme, $D_{2}\left(\mu_{R}\right)$ will be calculated as follows. First $m_{\pi}^{2} / \epsilon$ poles are subtracted. The finite counterterms are then determined by including graphs with a single $D_{2}\left(\mu_{R}\right)$ or potential pion and any number of $C_{0}\left(\mu_{R}\right)$ vertices in $\mathcal{A}^{s}\left(D_{2}\right)$. Contributions from these graphs that are nonanalytic in $m_{\pi}^{2}$ are dropped.

There is a $m_{\pi}^{2} / \epsilon$ pole in the $\mathcal{O}\left(Q^{0}\right)$ graph in the third row and third column of Fig. 4 [2,3]; so we have a counterterm

$$
\delta^{2, \mathrm{uv}} D_{2}=-i\left(\frac{M C_{0}^{\text {finite }}}{4 \pi}\right)^{2} \frac{g_{A}^{2}}{4 f^{2}}\left[\frac{1}{2 \epsilon}-\gamma+\log (\pi)\right] .
$$

Note that when this counterterm is dressed with $C_{0}$ bubbles the extra factors of $2-\ln 2$ from Eq. (4.3) will cancel without the need for an additional finite term in $\delta^{2, \text { uv }} D_{2}$. After subtracting this counterterm the value of the two-loop graph is

$$
\begin{aligned}
& i\left(\frac{M C_{0}^{\text {finite }}}{4 \pi}\right)^{2} \frac{g_{A}^{2}}{2 f^{2}}\left[-(i p)^{2}+\frac{m_{\pi}^{2}}{2}+\frac{m_{\pi}^{2}}{2} \ln \left(\frac{\mu^{2}}{m_{\pi}^{2}}\right)\right. \\
& \left.\quad-m_{\pi}^{2} \ln \left(1-\frac{2 i p}{m_{\pi}}\right)\right] .
\end{aligned}
$$

For the PDS scheme we set $\mu=\mu_{R}$ and then find the finite counterterms

$$
\begin{aligned}
\delta^{1} D_{2}\left(\mu_{R}\right)= & 2\left(\frac{M \mu_{R}}{4 \pi}\right) C_{0}\left(\mu_{R}\right) D_{2}\left(\mu_{R}\right) \\
\delta^{n} D_{2}\left(\mu_{R}\right)= & (-1)^{n+1}\left[(n+1)\left(\frac{M \mu_{R}}{4 \pi}\right)^{n} C_{0}\left(\mu_{R}\right)^{n} D_{2}\left(\mu_{R}\right)\right. \\
& -\frac{(n-1)}{2}\left(\frac{M \mu_{R}}{4 \pi}\right)^{n-2} C_{0}\left(\mu_{R}\right)^{n}\left(\frac{M}{4 \pi}\right)^{2} \frac{g_{A}^{2}}{2 f^{2}} \\
& \left.\times \ln \left(\frac{\mu_{R}^{2}}{\mu_{0}^{2}}\right)\right] \quad \text { for } n \geqslant 2 .
\end{aligned}
$$

Here $\mu_{0}$ is an unknown scale expected to be of order $\Lambda_{\pi}$.

In the OS scheme, the $\delta^{1} D_{2}\left(\mu_{R}\right)$ counterterm is the same as in the PDS scheme. In dimensional regularization logarithms of the form $\ln \left(\mu^{2} / m_{\pi}^{2}\right)$ will appear in loop graphs. To make the $\mu^{2}$-dependent part analytic in $m_{\pi}^{2}$ we write

$$
\ln \left(\frac{\mu^{2}}{m_{\pi}^{2}}\right)=\ln \left(\frac{\mu^{2}}{\mu_{R}^{2}}\right)+\ln \left(\frac{\mu_{R}^{2}}{m_{\pi}^{2}}\right),
$$

and then subtract the $\ln \left(\mu^{2} / \mu_{R}^{2}\right)$ term with the counterterms. This will give $D_{2}\left(\mu_{R}\right)$ a $\mu_{R}$ dependence which cancels the $\ln \left(\mu_{R}^{2} / m_{\pi}^{2}\right)$ in the amplitude. In the OS scheme, the $m_{\pi}^{2} / 2$ in Eq. (5.3) gets subtracted along with the logarithm. We find

$$
\begin{aligned}
\delta^{n} D_{2}\left(\mu_{R}\right)= & (-1)^{n+1}\left\{(n+1)\left(\frac{M \mu_{R}}{4 \pi}\right)^{n} C_{0}\left(\mu_{R}\right)^{n} D_{2}\left(\mu_{R}\right)\right. \\
& -\frac{(n-1)}{2}\left(\frac{M \mu_{R}}{4 \pi}\right)^{n-2} C_{0}\left(\mu_{R}\right)^{n}\left(\frac{M}{4 \pi}\right)^{2} \frac{g_{A}^{2}}{2 f^{2}} \\
& \left.\times\left[-1+\ln \left(\frac{\mu_{R}^{2}}{\mu^{2}}\right)\right]\right\} \quad \text { for } n \geqslant 2 .
\end{aligned}
$$

Summing the counterterms the solutions for $D_{2}\left(\mu_{R}\right)$ are then 


$$
\frac{D_{2}^{(s)}\left(\mu_{R}\right)}{C_{0}^{(s)}\left(\mu_{R}\right)^{2}}=\frac{D_{2}^{\text {finite }}}{\left(C_{0}^{\text {finite }}\right)^{2}}+\frac{M}{8 \pi}\left(\frac{M g_{A}^{2}}{8 \pi f^{2}}\right) \ln \left(\frac{\mu_{R}^{2}}{\mu_{0}^{2}}\right)
$$

in the PDS scheme,

$$
\frac{D_{2}^{(s)}\left(\mu_{R}\right)}{C_{0}^{(s)}\left(\mu_{R}\right)^{2}}=\frac{D_{2}^{\text {finite }}}{\left(C_{0}^{\text {finite }}\right)^{2}}+\frac{M}{8 \pi}\left(\frac{M g_{A}^{2}}{8 \pi f^{2}}\right)\left[-1+\ln \left(\frac{\mu_{R}^{2}}{\mu^{2}}\right)\right]
$$

in the OS scheme,

which can be written as

$$
\frac{D_{2}^{(s)}\left(\mu_{R}\right)}{C_{0}^{(s)}\left(\mu_{R}\right)^{2}}=\frac{M}{8 \pi}\left(\frac{M g_{A}^{2}}{8 \pi f^{2}}\right) \ln \left(\frac{\mu_{R}^{2}}{\tilde{\mu}^{2}}\right)
$$

where

$$
\begin{aligned}
& \tilde{\mu}^{2}=\mu_{0}^{2} \exp \left(\frac{-64 \pi^{2} f^{2} D_{2}^{\text {finite }}}{M^{2} g_{A}^{2}\left(C_{0}^{\text {finite }}\right)^{2}}\right) \quad \text { in the PDS scheme, } \\
& \tilde{\mu}^{2}=\mu^{2} \exp \left(1-\frac{64 \pi^{2} f^{2} D_{2}^{\text {finite }}}{M^{2} g_{A}^{2}\left(C_{0}^{\text {finite }}\right)^{2}}\right) \quad \text { in the OS scheme. }
\end{aligned}
$$

The parameter $\tilde{\mu}$ must be determined by fitting to the data. With $m_{\pi} \sim Q \sim \mu_{R}, D_{2}\left(\mu_{R}\right) m_{\pi}^{2} \sim Q^{0}$ in both the OS and PDS schemes, implying that $D_{2}\left(\mu_{R}\right)$ should be treated perturbatively.

\section{SCHEMES AND AMPLITUDES}

In this section, the amplitudes in the ${ }^{1} S_{0}$ and ${ }^{3} S_{1}$ channels are presented to order $Q^{0}$, both in the PDS [2,3] and OS schemes. Fits to the ${ }^{1} S_{0}$ and ${ }^{3} S_{1}$ phase shift data are done in both schemes for different values of $\mu_{R}$. As pointed out in Ref. [17], one has the freedom to split $C_{0}\left(\mu_{R}\right)$ into perturbative and nonperturbative pieces: $C_{0}\left(\mu_{R}\right)=C_{0}^{\mathrm{np}}\left(\mu_{R}\right)$ $+C_{0}^{\mathrm{p}}\left(\mu_{R}\right)$, where $C_{0}^{\mathrm{np}}\left(\mu_{R}\right) \sim Q^{-1}$ and $C_{0}^{\mathrm{p}}\left(\mu_{R}\right) \sim Q^{0}$. This division is necessary in the PDS scheme in order to obtain $\mu_{R}$-independent amplitudes at each order. Furthermore, $C_{0}^{\mathrm{np}}\left(\mu_{R}\right) \sim 1 / \mu_{R}$, and so the coefficients scale in a manner consistent with the power counting for all $\mu_{R}>1 / a$. For convenience we will drop the superscript "np" in what follows. Some issues that arise in matching the pion theory onto the effective range expansion are also discussed.

First, we give the nucleon-nucleon scattering amplitudes in the PDS and OS schemes. In the PDS scheme, the amplitudes were calculated to order $Q^{0}$ in Refs. [2,3]. At this order, amplitudes in the ${ }^{1} S_{0}$ and ${ }^{3} S_{1}$ channels have the same functional form

$$
A=A^{(-1)}+A^{(0, a)}+A^{(0, b)}+\mathcal{O}\left(Q^{1}\right) .
$$

In both the OS and PDS schemes we have

$$
\begin{aligned}
A^{(-1)}= & -\frac{4 \pi}{M} \frac{1}{4 \pi /\left[M C_{0}\left(\mu_{R}\right)\right]+\mu_{R}+i p}, \\
\frac{A^{(0, a)}}{\left[A^{(-1)}\right]^{2}}= & \frac{g_{A}^{2} m_{\pi}^{2}}{2 f^{2}}\left(\frac{M}{4 \pi}\right)^{2}\left\{\frac{1}{2} \ln \left(\frac{\mu_{R}^{2}}{m_{\pi}^{2}}\right)\right. \\
& -\left(\frac{4 \pi}{M C_{0}\left(\mu_{R}\right)}+\mu_{R}\right) \frac{1}{p} \tan ^{-1}\left(\frac{2 p}{m_{\pi}}\right) \\
& \left.+\left[\left(\frac{4 \pi}{M C_{0}\left(\mu_{R}\right)}+\mu_{R}\right)^{2}-p^{2}\right] \frac{1}{4 p^{2}} \ln \left(1+\frac{4 p^{2}}{m_{\pi}^{2}}\right)\right\} \\
& -\frac{D_{2}\left(\mu_{R}\right) m_{\pi}^{2}}{C_{0}\left(\mu_{R}\right)^{2}} .
\end{aligned}
$$

The remaining part of the order $Q^{0}$ PDS amplitude is

$$
\begin{aligned}
\frac{A^{(0, b)}}{\left[A^{(-1)}\right]^{2}}= & -\frac{C_{2}\left(\mu_{R}\right) p^{2}}{C_{0}\left(\mu_{R}\right)^{2}}+\frac{1}{2} \frac{g_{A}^{2} m_{\pi}^{2}}{2 f^{2}}\left(\frac{M}{4 \pi}\right)^{2} \\
& -\frac{1}{C_{0}\left(\mu_{R}\right)^{2}}\left[\frac{g_{A}^{2}}{2 f^{2}}+C_{0}^{\mathrm{p}}\left(\mu_{R}\right)\right] .
\end{aligned}
$$

Note that since we have made a different finite subtraction than $\mathrm{KSW}$, the second term has a prefactor of $1 / 2$, rather than a 1 as in Ref. [3]. In the OS scheme,

$$
\begin{aligned}
\frac{A^{(0, b)}}{\left[A^{(-1)}\right]^{2}}= & -\frac{C_{2}\left(\mu_{R}\right) p^{2}}{C_{0}\left(\mu_{R}\right)^{2}}-\frac{g_{A}^{2}}{2 f^{2}}\left(1+\frac{p^{2}}{\mu_{R}^{2}}\right) \\
& \times\left(\frac{1}{C_{0}\left(\mu_{R}\right)}+\frac{M \mu_{R}}{4 \pi}\right)^{2}-\frac{C_{0}^{\mathrm{p}}\left(\mu_{R}\right)}{C_{0}\left(\mu_{R}\right)^{2}} .
\end{aligned}
$$

In Appendix B, we give relations between the OS and PDS couplings that appear at this order. Using these equations it is easy to verify that the amplitudes are equivalent in the two schemes.

In the PDS scheme, there are order $Q^{0}$ contributions to $\beta_{0}$ [cf. Eq. (4.10)]. If the order $Q^{0}$ contributions are separated from the order $1 / Q$ pieces, the beta function for $C_{0}^{\mathrm{p}}\left(\mu_{R}\right)$ is

$$
\mu_{R} \frac{\partial C_{0}^{\mathrm{p}}\left(\mu_{R}\right)}{\partial \mu_{R}}=2 \frac{M \mu_{R}}{4 \pi} C_{0}\left(\mu_{R}\right)\left[C_{0}^{\mathrm{p}}\left(\mu_{R}\right)+\frac{g_{A}^{2}}{2 f^{2}}\right]+\mathcal{O}(Q) .
$$

This equation has the solution

$$
\frac{C_{0}^{\mathrm{p}}\left(\mu_{R}\right)}{C_{0}\left(\mu_{R}\right)^{2}}=\frac{M}{4 \pi} K-\frac{g_{A}^{2}}{2 f^{2}} \frac{1}{C_{0}\left(\mu_{R}\right)^{2}},
$$

where $K$ is a constant which must be order $Q^{2}$ for $C_{0}^{\mathrm{p}}\left(\mu_{R}\right)$ $\sim Q^{0}$. [Recall, from Eq. (3.14), that $K=\gamma-1 / a \lesssim 1 / a$ in the pure nucleon theory.] Including $C_{0}^{\mathrm{p}}\left(\mu_{R}\right)$ makes the PDS amplitudes explicitly $\mu_{R}$ independent. In performing fits to the data the constant $K$ and coupling $D_{2}\left(\mu_{R}\right)$ cannot be determined independently. In what follows we will drop $K$ and simply remember that the values of $D_{2}\left(\mu_{R}\right)$ extracted from the fits may differ from the renormalized coupling in the 
TABLE I. ${ }^{1} S_{0}$ and ${ }^{3} S_{1}$ couplings in the PDS scheme. $C_{0}\left(\mu_{R}\right)$ (in $\mathrm{fm}^{2}$ ), $C_{2}\left(\mu_{R}\right)$ (in $\mathrm{fm}^{4}$ ), and $D_{2}\left(\mu_{R}\right)$ (in $\mathrm{fm}^{4}$ ) are fit to the Nijmegen data at different values of $\mu_{R}$.

\begin{tabular}{rrrrrrr}
\hline \hline \multicolumn{3}{l}{$\mu_{R}$} & \multicolumn{3}{c}{ Fit to ${ }^{1} S_{0}$} & \multicolumn{3}{c}{ Fit to ${ }^{3} S_{1}$} \\
$(\mathrm{MeV})$ & $C_{0}\left(\mu_{R}\right)$ & $C_{2}\left(\mu_{R}\right)$ & $D_{2}\left(\mu_{R}\right)$ & $C_{0}\left(\mu_{R}\right)$ & $C_{2}\left(\mu_{R}\right)$ & $D_{2}\left(\mu_{R}\right)$ \\
\hline 70 & -6.48 & 10.11 & -0.532 & -22.73 & 171.0 & -70.41 \\
100 & -4.71 & 5.36 & 1.763 & -9.93 & 32.7 & -4.157 \\
137 & -3.53 & 3.01 & 2.000 & -5.88 & 11.5 & 1.500 \\
160 & -3.05 & 2.25 & 1.869 & -4.69 & 7.32 & 1.897 \\
280 & -1.79 & 0.772 & 1.105 & -2.19 & 1.57 & 1.004 \\
\hline \hline
\end{tabular}

Lagrangian. In the PDS scheme, if $C_{0}^{\mathrm{p}}\left(\mu_{R}\right)$ is omitted from our expressions, then $D_{2}\left(\mu_{R}\right)$ does not follow the renormalization group equation, as we will see below.

In the OS scheme, the constant $g_{A}^{2} /\left(2 f^{2}\right)$ in Eqs. (6.5) and (6.6) is not present; so $C_{0}^{\mathrm{p}}\left(\mu_{R}\right) / C_{0}\left(\mu_{R}\right)^{2}$ is $\mu_{R}$ independent. The OS amplitudes $A^{(-1)}$ and $A^{(0)}$ are therefore $\mu_{R}$ independent without $C_{0}^{\mathrm{p}}\left(\mu_{R}\right)$ as can be seen by examining Eqs. (4.13) and (5.7). In the OS scheme the constant $K$ will also be absorbed into $D_{2}\left(\mu_{R}\right)$.

Using the Nijmegen phase shifts [25] between 7 and 100 $\mathrm{MeV}$, we fit the coefficients $C_{0}\left(\mu_{R}\right), C_{2}\left(\mu_{R}\right)$, and $D_{2}\left(\mu_{R}\right)$. We took $m_{\pi}=137 \mathrm{MeV}$. Clearly we would like to bias the fit towards the low momentum points since that is where the theoretical error is smallest. This can be accomplished by assigning a percent error, $\simeq p /(300 \mathrm{MeV})$, to the data and then minimizing the $\chi^{2}$ function. In Tables I and II we show the values ${ }^{4}$ of $C_{0}\left(\mu_{R}\right), C_{2}\left(\mu_{R}\right)$, and $D_{2}\left(\mu_{R}\right)$ extracted from the fits for $\mu_{R}=70,100,137,160,280 \mathrm{MeV}$. These values exhibit the $\mu_{R}$ dependence predicted by the RGE's to $\sim 1 \%$ in the ${ }^{1} S_{0}$ channel and $\sim 4 \%$ in the ${ }^{3} S_{1}$ channel. In Fig. 6 the results of the fits are shown. The results of the fits shown in the figure are identical in both schemes. Higher order corrections will give contributions to $\delta$ of the form $p^{2} / \Lambda_{\pi}^{2}$. The error in $\delta$ at $p=300 \mathrm{MeV}$ is consistent with $\Lambda_{\pi}$ $\gtrsim 500 \mathrm{MeV}$.

For processes involving the deuteron it is convenient to fix $C_{0}\left(\mu_{R}\right)$ using the deuteron binding energy, $C_{0}\left(m_{\pi}\right)=$ $-5.708 \mathrm{fm}^{2}$. With this constraint we find $C_{2}\left(m_{\pi}\right)$ $=10.80 \mathrm{fm}^{4}$ and $D_{2}\left(m_{\pi}\right)=1.075 \mathrm{fm}^{4}$ in the PDS scheme. The fit to the phase shift data with these values is as good as that in Fig. 6.

In the PDS scheme, it is necessary to break $C_{0}\left(\mu_{R}\right)$ into perturbative and nonperturbative parts to obtain amplitudes that are $\mu_{R}$ independent order by order. If $C_{0}^{\mathrm{p}}\left(\mu_{R}\right)$ is omitted, then the values of $D_{2}\left(\mu_{R}\right)$ determined from the fit will not follow the RGE. To see this we define the $\mu_{R}$-independent quantity

${ }^{4}$ The coefficients extracted from our fits differ from those in Ref. [3] because we have emphasized the low energy data as opposed to doing a global fit. It is interesting to note that using our PDS value $C_{2}\left(\mu_{R}=137 \mathrm{MeV}\right)=11.5 \mathrm{fm}^{4}$, the prediction for the rms charge radius of the deuteron [21] becomes $1.966 \mathrm{fm}$ which is within $1 \%$ of the experimental result.
TABLE II. ${ }^{1} S_{0}$ and ${ }^{3} S_{1}$ couplings in the OS scheme. $C_{0}\left(\mu_{R}\right)$ (in $\mathrm{fm}^{2}$ ), $C_{2}\left(\mu_{R}\right)$ (in $\mathrm{fm}^{4}$ ), and $D_{2}\left(\mu_{R}\right)$ (in $\mathrm{fm}^{4}$ ) are fit to the Nijmegen data at different values of $\mu_{R}$.

\begin{tabular}{rcccccc}
\hline \hline \multirow{2}{*}{$\mu_{R}$} & \multicolumn{3}{c}{ Fit to ${ }^{1} S_{0}$} & \multicolumn{3}{c}{ Fit to ${ }^{3} S_{1}$} \\
$(\mathrm{MeV})$ & $C_{0}\left(\mu_{R}\right)$ & $C_{2}\left(\mu_{R}\right)$ & $D_{2}\left(\mu_{R}\right)$ & $C_{0}\left(\mu_{R}\right)$ & $C_{2}\left(\mu_{R}\right)$ & $D_{2}\left(\mu_{R}\right)$ \\
\hline 70 & -6.50 & 9.75 & -6.047 & -24.1 & 121.0 & -170.1 \\
100 & -4.73 & 5.33 & -1.143 & -10.0 & 27.3 & -20.18 \\
137 & -3.54 & 3.00 & 0.378 & -5.92 & 10.5 & -4.124 \\
160 & -3.06 & 2.25 & 0.658 & -4.74 & 6.89 & -1.671 \\
280 & -1.80 & 0.779 & 0.692 & -2.23 & 1.61 & 0.2985 \\
\hline \hline
\end{tabular}

$$
R=c\left[\frac{-D_{2}\left(\mu_{R}\right)}{C_{0}\left(\mu_{R}\right)^{2}}+\frac{M}{8 \pi}\left(\frac{M g_{A}^{2}}{8 \pi f^{2}}\right) \ln \left(\frac{\mu_{R}^{2}}{\mu_{0}^{2}}\right)\right],
$$

and choose the constant $c$ so that $R=1$ for $\mu_{R}=137 \mathrm{MeV}$. For other values of $\mu_{R}$ the deviation of $R$ from 1 gives the discrepancy between the values predicted by the RGE and those extracted from the fit. For $\mu_{R}=70,280 \mathrm{MeV}$ we find $R=-0.53,7.25$ in the ${ }^{1} S_{0}$ channel and $R=-0.52,11.4$ in the ${ }^{3} S_{1}$ channel. These large deviations disappear if $C_{0}^{\mathrm{p}}\left(\mu_{R}\right)$ is included. Without $C_{0}^{\mathrm{p}}\left(\mu_{R}\right)$, the PDS amplitude is still $\mu_{R}$ independent to the order that one is working in. However, as explained below, this residual $\mu_{R}$ dependence gives larger corrections than expected [14] since it makes the tuning that was set up to give the large scattering length $\mu_{R}$ dependent.

For momenta $p \ll m_{\pi}$ the pion can be integrated out, leaving the effective range expansion

$$
p \cot (\delta)=-\frac{1}{a}+\frac{r_{0}}{2} p^{2}+v_{2} p^{4}+v_{3} p^{6}+v_{4} p^{8}+\cdots
$$

Performing a matching calculation between the two theories gives expressions for $a, r_{0}$, and $v_{i}$ in terms of the parameters in the pion theory. Since the theory with pions is an expansion in $Q$, these predictions take the form of Taylor series in $Q / \Lambda_{\pi}$ :

$$
\frac{1}{a}=\gamma+\sum_{i=2}^{\infty} B_{0}^{(i)}, \quad \frac{r_{0}}{2}=\sum_{i=0}^{\infty} B_{1}^{(i)}, \quad v_{n}=\sum_{i=2-2 n}^{\infty} B_{n}^{(i)},
$$

where $B_{n}^{(i)} \sim Q^{i}$. At this time only the first coefficient in each series is known since $p \cot \delta$ has only been calculated to order $Q^{2}$. The notation

$$
\gamma=\frac{4 \pi}{M C_{0}\left(\mu_{R}\right)}+\mu_{R}
$$

will be used to denote the location of the perturbative pole in the amplitudes. In the PDS scheme, 


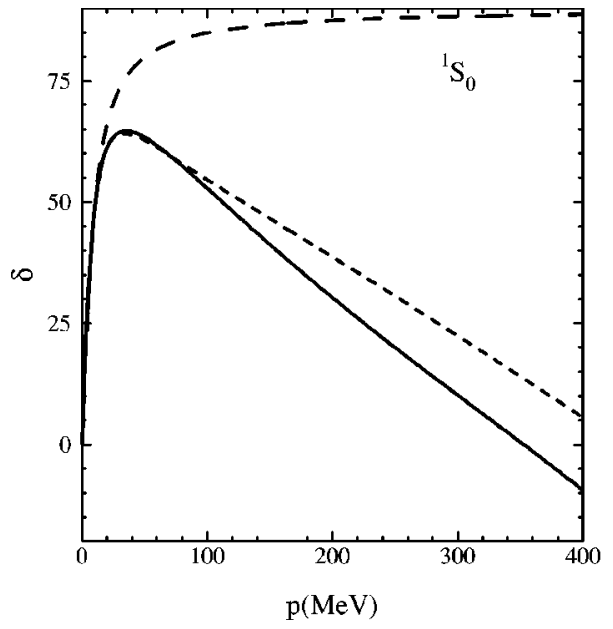

$$
\begin{aligned}
B_{0}^{(2)}= & \left(\frac{-4 \pi}{M}\right) \frac{m_{\pi}^{2} D_{2}\left(\mu_{R}\right)}{C_{0}\left(\mu_{R}\right)^{2}} \\
& +\frac{m_{\pi}^{2} M g_{A}^{2}}{8 \pi f^{2}}\left[\frac{1}{2}+\frac{1}{2} \ln \left(\frac{\mu_{R}^{2}}{m_{\pi}^{2}}\right)-\frac{2 \gamma}{m_{\pi}}+\frac{\gamma^{2}}{m_{\pi}^{2}}\right]-K, \\
B_{1}^{(0)}= & \left(\frac{4 \pi}{M}\right) \frac{C_{2}\left(\mu_{R}\right)}{\left[C_{0}\left(\mu_{R}\right)\right]^{2}}+\frac{M g_{A}^{2}}{8 \pi f^{2}}\left[1-\frac{8 \gamma}{3 m_{\pi}}+\frac{2 \gamma^{2}}{m_{\pi}^{2}}\right] .
\end{aligned}
$$

Note that if $C_{0}^{\mathrm{p}}\left(\mu_{R}\right)$ had been neglected, then $B_{0}^{(2)}$ would not be $\mu_{R}$ independent. With $\mu_{R}=m_{\pi}$, Eq. (6.11) agrees with Ref. [3] if their definition of $D_{2}\left(\mu_{R}\right)$ is adopted. In the OS scheme we have

$$
\begin{aligned}
B_{0}^{(2)}= & \left(\frac{-4 \pi}{M}\right) \frac{m_{\pi}^{2} D_{2}\left(\mu_{R}\right)}{C_{0}\left(\mu_{R}\right)^{2}} \\
& +\frac{m_{\pi}^{2} M g_{A}^{2}}{8 \pi f^{2}}\left[\frac{1}{2} \ln \left(\frac{\mu_{R}^{2}}{m_{\pi}^{2}}\right)-\frac{2 \gamma}{m_{\pi}}\right]-K, \\
B_{1}^{(0)}= & \left(\frac{4 \pi}{M}\right) \frac{C_{2}\left(\mu_{R}\right)}{C_{0}\left(\mu_{R}\right)^{2}}+\frac{M g_{A}^{2}}{8 \pi f^{2}}\left[\frac{\gamma^{2}}{\mu_{R}^{2}}+1-\frac{8 \gamma}{3 m_{\pi}}+\frac{2 \gamma^{2}}{m_{\pi}^{2}}\right] .
\end{aligned}
$$

The values of the remaining $B_{n}^{(i)}$ determined at this order are the same in both schemes:

$$
B_{n}^{(2-2 n)}=\frac{M g_{A}^{2}}{2 \pi f^{2}}\left(\frac{-4}{m_{\pi}^{2}}\right)^{n-1}\left[\frac{1}{4 n}-\frac{2 \gamma}{(2 n+1) m_{\pi}}+\frac{\gamma^{2}}{(n+1) m_{\pi}^{2}}\right] .
$$

FIG. 6. Fit to the phase shift data emphasizing the low momentum region. The solid line is the Nijmegen fit to the data [25], the long dashed line is the order $1 / Q$ result, and the short dashed line is the order $Q^{0}$ result.
For $n=2,3,4$, Eq. (6.13) gives the low energy theorems derived in Ref. [16] if we set $\gamma=1 / a$.

Recall that the unnaturally large scattering length $a$ is a fine-tuning that was accounted for by demanding that, in Eq. (6.10), $C_{0}\left(\mu_{R}\right)$ be close to its ultraviolet fixed point and $\gamma$ $\approx 1 / a$. Examining the expression for $1 / a$ in Eq. (6.9) it may seem that this could be destroyed by chiral corrections. If $D_{2}\left(\mu_{R}\right) \sim C_{0}\left(\mu_{R}\right)^{2}$, then the first term gives $B_{0}^{(2)}$ $\sim 205 \mathrm{MeV}$. In fact, from Table III, we see that the fit gives $B_{0}^{(2)} \lesssim 1 / a$. The reason for this small value is that since $A^{(0)}$ $\propto\left(A^{(-1)}\right)^{2}$ the amplitude has a double pole. Since this pole is spurious (occurring from the perturbative expansion), the residue of the double pole must be small in order to fit the data. This leads to a good fit condition [17] which will be approximately satisfied:

$$
\left.\frac{A^{(0)}}{\left[A^{(-1)}\right]^{2}}\right|_{-i p=\gamma}=0
$$

As explained in Ref. [17], this condition implies $B_{0}^{(2)}$ $\simeq 4 \pi \gamma^{2} / M$. In fact this gives the right order of magnitude for the values of $B_{0}^{(2)}$ determined from the fits in Table III. Similar good fit conditions occur at higher order, keeping the coefficients $B_{0}^{(i)}$ small. Thus the tuning $\gamma \approx 1 / a$ is not destroyed, but instead naturally kept by the form of the perturbative expansion. The division of $C_{0}\left(\mu_{R}\right)$ into nonperturbative and perturbative pieces is arbitrary, allowing us to set up the theory so that the $Q$ expansion for $1 / a$ is well behaved.

In Table III we see that when $B_{0}^{(2)}$ is added to $\gamma$, values of $1 / a$ are obtained which are close to the physical values, $1 / a\left({ }^{1} S_{0}\right)=-8.32 \mathrm{MeV}$ and $1 / a\left({ }^{3} S_{1}\right)=36.4 \mathrm{MeV}$. It is encouraging that the values of $\gamma$ found from fits in the ${ }^{3} S_{1}$ channel are close to the physical pole in the amplitude which

TABLE III. Values of $\gamma, B_{0}^{(2)}, 1 / a$, and $r_{0}$ (in MeV) obtained from our fits. Three values of $\mu_{R}$ are shown to emphasize that the value of the extracted parameters depends weakly on $\mu_{R}$.

\begin{tabular}{lcccccccc}
\hline \hline & \multicolumn{3}{c}{${ }^{1} S_{0}$ fit } & \multicolumn{4}{c}{${ }^{3} S_{1}$ fit } \\
$\mu_{R}(\mathrm{MeV})$ & $\gamma$ & $B_{0}^{(2)}$ & $1 / a$ & $r_{0}$ & $\gamma$ & $B_{0}^{(2)}$ & $1 / a$ & $r_{0}$ \\
\hline 70 & -10.18 & 2.05 & -8.124 & 0.01468 & 48.39 & -15.82 & 32.57 & 0.01101 \\
137 & -10.16 & 2.04 & -8.121 & 0.01480 & 48.96 & -16.76 & 32.19 & 0.01098 \\
280 & -10.23 & 2.12 & -8.105 & 0.01484 & 46.39 & -12.64 & 33.76 & 0.01111 \\
\hline \hline
\end{tabular}




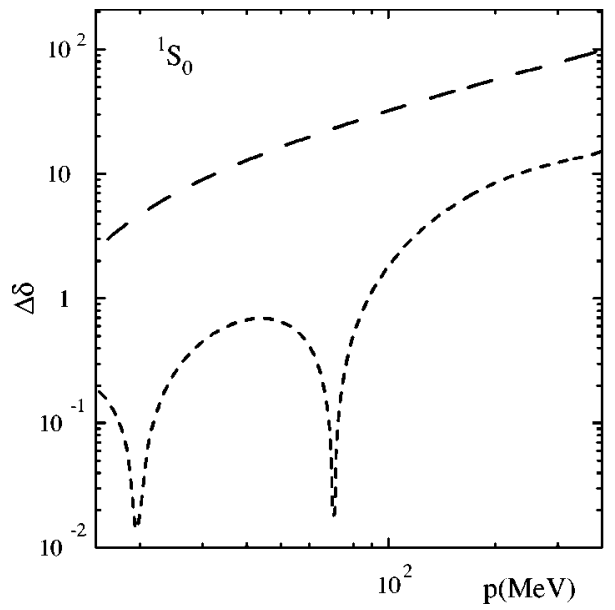

corresponds to the deuteron, $\gamma=45.7 \mathrm{MeV}$. Values for $r_{0}$ can also be predicted from the fits using Eq. (6.12). Experimentally, $\quad r_{0}\left({ }^{1} S_{0}\right)=0.0139 \mathrm{MeV}^{-1}$ and $r_{0}\left({ }^{3} S_{1}\right)$ $=0.00888 \mathrm{MeV}^{-1}$; so the values in Table III agree to the expected accuracy. It is not yet clear [17] whether values of the $v_{i}$ extracted from experimental data $[16,26]$ are accurate enough to test the low energy theorems for $v_{2}, v_{3}$, and $v_{4}$.

\section{DETERMINING THE RANGE $\Lambda_{\pi}$}

Here we will examine the phase shift data to see what it tells us about the range of the effective field theory with perturbative pions. In Ref. [15], a Lepage analysis is performed on the observable $p \cot \delta(p)$ in the ${ }^{1} S_{0}$ channel. Near $350 \mathrm{MeV}$ the experimental ${ }^{1} S_{0}$ phase shift passes through zero. Therefore, the error $\left|p \cot \delta^{\mathrm{NPWA}}-p \cot \delta^{\mathrm{EFT}}\right|$ is greatly exaggerated since $p \cot \delta(p) \rightarrow \infty$. To avoid this problem we will use the ${ }^{1} S_{0}$ and ${ }^{3} S_{1}$ phase shifts as our observables, since $\Delta \delta=\left|\delta^{\mathrm{NPWA}}-\delta^{\mathrm{EFT}}\right|$ remains finite for all $p$. The nextto-leading order amplitudes given in Sec. V will be used. The phase shifts have an expansion of the form $\delta=\delta^{(0)}$ $+\delta^{(1)}+\mathcal{O}\left(Q^{2} /^{2}\right)$, where [3]

$$
\begin{gathered}
\delta^{(0)}=-\frac{i}{2} \ln \left[1+i \frac{p M}{2 \pi} \mathcal{A}^{(-1)}\right], \\
\delta^{(1)}=\frac{p M}{4 \pi} \frac{\mathcal{A}^{(0)}}{1+i p M /(2 \pi) \mathcal{A}^{(-1)}} .
\end{gathered}
$$

Recall that a momentum expansion of $\delta$ would result in terms with only odd powers of $p$. However, the expansion for $\delta$ in Eq. (7.1) is not simply a momentum expansion; so the next-to-leading order calculation can have errors which scale as $p^{2} / \Lambda_{\pi}^{2}$. For example, once pions are included we can have a term $p^{2} \tan ^{-1}\left(2 p / m_{\pi}\right)$ which is odd in $p$, order $Q^{2}$, and scales as $p^{2}$ for large momenta.

In Fig. 7, we plot $\Delta \delta$ versus $p$ using log-log axes. Note that the sharp dips in Fig. 7 are just locations where the theory happens to agree with the data exactly. The Nijmegen data [25] are available up to $p=405 \mathrm{MeV}$. In a theory with just a momentum expansion the errors will appear as straight lines on the log-log plot as pointed out by Lepage [6]. In the pion theory the expansion is in both $m_{\pi} / \Lambda_{\pi}$ and $p / \Lambda_{\pi}$; so this is no longer true. For $p>m_{\pi}$ we expect the errors to be of the form ${ }^{5}$

$$
\begin{aligned}
\Delta \delta^{(0)} \sim\left(1+\frac{m_{\pi}}{\Lambda_{\pi}}+\ldots\right) \frac{p}{\Lambda_{\pi}}+\cdots \\
\Delta \delta^{(1)} \sim\left(\frac{m_{\pi}}{\Lambda_{\pi}}+\frac{m_{\pi}^{2}}{\Lambda_{\pi}^{2}}+\ldots\right) \frac{p}{\Lambda_{\pi}} \\
+\left(1+\frac{m_{\pi}}{\Lambda_{\pi}}+\ldots\right) \frac{p^{2}}{\Lambda_{\pi}^{2}}+\cdots
\end{aligned}
$$

The fact that there is always a $p / \Lambda_{\pi}$ error arises from the fact that, as seen in Eq. (6.9), $r_{0}$ is reproduced in the effective field theory as an expansion in $m_{\pi} / \Lambda_{\pi}$. For $p / \Lambda_{\pi}$ $\gg m_{\pi} / \Lambda_{\pi}$ the slope of the lines on the plot should indicate the lowest power of $p$ that has not been included. At low momentum the error in $\Delta \delta^{(n)}$ is dominated by the $p m_{\pi}^{n} / \Lambda_{\pi}^{n+1}$ term and the lines should be parallel. From Fig. 7 we see that the error is smallest at low momentum and increases as the momentum increases, which is how the theoretical error is expected to behave.

It is clear that even for $p \sim 400 \mathrm{MeV}$ the next-to-leading order calculations reduce the error in the phase shift. Because two new parameters are added at next-to-leading order, it is always possible to force exact agreement at some value of $p$. However, if one were to force the data to agree too well at high momentum, then this would destroy the agreement at low momentum. Since the improvement of the fit in Fig. 7 at high momentum does not come at the expense of the fit at low momentum, this is evidence that the error is being reduced in a systematic way. At high momentum one expects that the error is $\sim p^{2} / \Lambda_{\pi}^{2}$. From Fig. 7, $\Delta \delta \sim 0.26 \mathrm{rad}$ for $p=400 \mathrm{MeV}$, implying $\Lambda_{\pi} \sim 800 \mathrm{MeV}$. This is only a rough estimate for the range because we cannot yet exclude the possibility that the next-to-next-to-leading order phase shift has an anomalously small coefficient. Even though the

\footnotetext{
${ }^{5}$ At momenta $1 / a \ll p \ll m_{\pi}$ we could have $\Delta \delta^{(0)} \sim B_{0}^{(2)} / p$ $\sim m_{\pi}^{2} /\left(\Lambda_{\pi} p\right)$. However, as explained in Sec. VI, $B_{0}^{(2)} \lesssim 1 / a$, and so this term is very small.
} 
lines in Fig. 7 are not straight, they should still cross at approximately the range of the theory since at this point higher order corrections do not improve the agreement with the data. This error analysis is consistent with the possibility that the range is $\gtrsim 500 \mathrm{MeV}$.

Further information on the range of the effective field theory can be obtained by examining electromagnetic processes involving the deuteron [21,24], such as the deuteron charge radius, electromagnetic form factors, deuteron polarizability, and deuteron Compton scattering. For these observables errors are typically $\sim 30-40 \%$ at leading order and $\sim 10 \%$ at next-to-leading order. This is what one would expect if the expansion parameter $m_{\pi} / \Lambda_{\pi} \sim 1 / 3$, implying $\Lambda_{\pi}$ $\sim 410 \mathrm{MeV}$. This is consistent with our previous estimate for $\Lambda_{\pi}$. If the range is this large, one should expect that the error in deuteron properties will be at the few percent level once next-to-next-to-leading order calculations are performed.

\section{CONCLUSION}

In this paper the structure of the effective field theories of nucleons with and without pions is studied. We discuss a momentum subtraction scheme, the OS scheme, which obeys the KSW power counting. The method of local counterterms is used to obtain the renormalization group equations for the coupling constants in these theories. Using local counterterms defines the OS and PDS renormalization schemes unambiguously. Two-loop graphs with potential pions in the ${ }^{3} S_{1}$ channel are computed and shown to have $p^{2} / \epsilon$ poles. The presence of $1 / \epsilon$ poles implies that the only modelindependent piece of pion exchange is the part that can be treated perturbatively. We obtain the renormalized couplings $C_{0}\left(\mu_{R}\right), C_{2}\left(\mu_{R}\right)$, and $C_{4}\left(\mu_{R}\right)$ at order $Q$ in the OS and PDS schemes.

We have emphasized why it is important to have $\mu_{R}$-independent amplitudes order by order in $Q$. Such amplitudes are obtained automatically in the OS scheme. In the PDS scheme $\mu_{R}$-independent amplitudes may be obtained by treating part of $C_{0}\left(\mu_{R}\right)$ perturbatively. Another result concerns the large $\mu_{R}$ behavior of the couplings in this theory. In the OS scheme the coupling constants obey the KSW power counting for all $\mu_{R}>1 / a$. In the PDS scheme the breakdown in the power counting for $C_{0}\left(\mu_{R}\right)$ is avoided if $C_{0}\left(\mu_{R}\right)$ is split into nonperturbative and perturbative parts. Therefore, the breakdown of the scaling in the coupling constants is artificial.

Next-to-leading order calculations of nucleon-nucleon phase shift data [3] provide fits to data at large momenta which are far more accurate than one would expect if the theory broke down completely at $300 \mathrm{MeV}$. Of course, this does not mean that nucleon effective theory can be applied at arbitrarily high energies. The scale $M g_{A}^{2} /\left(8 \pi f^{2}\right)$ $\sim 300 \mathrm{MeV}$ is associated with short distance contributions from pion exchange and provides an order of magnitude estimate for the range. In the $S$-wave channel, $\Delta$ production and $\rho$ exchange become relevant at $\sim 700 \mathrm{MeV}$, which sets an upper limit on the range of the expansion. To get a better understanding of the range of the nucleon effective theory with perturbative pions one must examine experimental data. An error analysis of the $S$-wave phase shifts with next-to- leading-order calculations seems to be consistent with a range of $500 \mathrm{MeV}$. Though next-to-next-leading order corrections need to be compared with data and other processes investigated, we remain cautiously optimistic that the range could be as large as $500 \mathrm{MeV}$.

\section{ACKNOWLEDGEMENTS}

We would like to thank Mark Wise for many useful conversations. We also would like to thank H. Davoudiasl, S. Fleming, U. van Kolck, Z. Ligeti, S. Ouellette, and K. Scaldeferri for their comments. T.M. would like to acknowledge the hospitality of the Department of Physics at the University of Toronto, where part of this work was completed. This work was supported in part by the Department of Energy under Grant No. DE-FG03-92-ER 40701.

\section{APPENDIX A: LOOP INTEGRALS WITH A MOMENTUM CUTOFF REGULATOR}

Although the analysis in Sec. III used dimensional regularization to regulate divergent loop integrals, the results for the coefficients $C_{2 m}\left(\mu_{R}\right)$ in our momentum subtraction scheme are independent of this choice. As an exercise we will derive the counterterms for $C_{0}\left(\mu_{R}\right)$ and $C_{2}\left(\mu_{R}\right)$ using a momentum cutoff regulator $\Lambda$. This will give us the chance to see what type of complications can arise using a different regulator. Note that this is not the same as using a finite cutoff scheme. There the momentum cutoff plays a double role as both a regulator and as part of the subtraction scheme.

The graph in the first row first column of Fig. 3 gives

$$
\begin{aligned}
& i C_{0}^{2} M \int_{0}^{\Lambda} \frac{d^{3} q}{(2 \pi)^{3}} \frac{1}{\vec{q}^{2}-p^{2}} \\
& \quad=\frac{i M}{2 \pi^{2}} C_{0}^{2}\left[\Lambda+\frac{i \pi p}{2}-p \tanh ^{-1}\left(\frac{p}{\Lambda}\right)\right] \\
& =\frac{i M}{2 \pi^{2}} C_{0}^{2}\left[\Lambda+\frac{i \pi p}{2}-\frac{p^{2}}{\Lambda}-\frac{p^{4}}{3 \Lambda^{3}}-\cdots\right]
\end{aligned}
$$

An ultraviolet counterterm cancels the linear divergence,

$$
\delta^{1, \mathrm{uv}} C_{0}=\frac{M}{4 \pi}\left(C_{0}^{\text {finite }}\right)^{2}\left(-\frac{2 \Lambda}{\pi}\right) .
$$

and the same finite counterterm $\delta^{1} C_{0}\left(\mu_{R}\right)$ in Eq. (3.4) is used to satisfy the condition in Fig. 2. The renormalized graph is then the same as calculated in dimensional regularization in Sec. III. Note that contributions of order $p^{2}$ have been neglected in defining $C_{0}\left(\mu_{R}\right)$ as required by our renormalization condition. An added complication with a cutoff is that graphs with only $C_{0}$ 's give a contribution to the amplitude proportional to $p^{2}$. However, as $\Lambda \rightarrow \infty$, $p \tanh ^{-1}(p / \Lambda) \rightarrow 0$; so these terms can be completely neglected. This will remain true even for higher loops since the counterterms will always cancel dangerous powers of $\Lambda$ that appear in the numerator. At $n$ loops we find an ultraviolet counterterm of the form 


$$
\delta^{n, \mathrm{uv}} C_{0}=-\left(\frac{-M}{4 \pi}\right)^{n} C_{0}\left(\mu_{R}\right)^{n+1}\left(-\frac{2 \Lambda}{\pi}\right)^{n},
$$

while the finite counterterms are given by Eq. (3.4).

The graph in the third row first column of Fig. 3 gives

$$
\begin{aligned}
2 i C_{0} C_{2} \frac{M}{2} \int_{0}^{\Lambda} \frac{d^{3} q}{(2 \pi)^{3}} \frac{\vec{q}^{2}+p^{2}}{\vec{q}^{2}-p^{2}} \\
\quad=2 \frac{i M}{2 \pi^{2}} C_{0} C_{2}\left\{\frac{\Lambda^{3}}{6}+p^{2}\left[\Lambda+\frac{i \pi p}{2}-p \tanh ^{-1}\left(\frac{p}{\Lambda}\right)\right]\right\} .
\end{aligned}
$$

Note that there are different contributions from this graph when the vertices are in the order $C_{0} C_{0} C_{2}$ or $C_{0} C_{2} C_{0}$. At order $p^{2}$, this graph gives a correction to the counterterm $\delta^{1, \mathrm{uv}} C_{0}$, i.e., $\delta^{1, \mathrm{uv}} C_{0} \rightarrow \delta^{1, \mathrm{uv}} C_{0}+\delta^{1 * \text {,uv }} C_{0}$, where

$$
\delta^{1 *, \mathrm{uv}} C_{0}=-\frac{M}{4 \pi} 2 C_{0}^{\text {finite }} C_{2}^{\text {finite }} \frac{\Lambda^{3}}{3 \pi} .
$$

Unlike the contribution to $\delta^{1, \mathrm{uv}} C_{0}$ in Eq. (A3), $\delta^{1 * \text {, uv }} C_{0}$ is to be treated perturbatively, so that it only appears once in any graph. The justification of this fact is that this contribution to the counterterm appeared at order $Q^{0}$ (a purely formal trick to recover this counting is to take $\Lambda \sim \mu_{R} \sim Q$ ). The counterterm $\delta^{1, \text { uv }} C_{2}$ is fixed by considering the order $p^{2}$ terms in Fig. 3, row 3. From Eq. (A4) (the $\tanh ^{-1}$ piece can again be thrown away) we have

$$
\delta^{1, \mathrm{uv}} C_{2}=\frac{M}{4 \pi} 2 C_{0}^{\text {finite }} C_{2}^{\text {finite }}\left(-\frac{2 \Lambda}{\pi}\right) .
$$

The calculation for higher loops is similar and there are again corrections $\delta^{n *, \mathrm{uv}} C_{0}$ to $\delta^{n, \mathrm{uv}} C_{0}$ :

[1] S. Weinberg, Phys. Lett. B 251, 288 (1990); Nucl. Phys. B363, 3 (1991).

[2] D. B. Kaplan, M. J. Savage, and M. B. Wise, Phys. Lett. B 424, 390 (1998).

[3] D. B. Kaplan, M. J. Savage, and M. B. Wise, Nucl. Phys. B534, 329 (1998).

[4] U. van Kolck, nucl-th/9808007.

[5] C. Ordonez and U. van Kolck, Phys. Lett. B 291, 459 (1992); C. Ordonez, L. Ray, and U. van Kolck, Phys. Rev. Lett. 72, 1982 (1994); Phys. Rev. C 53, 2086 (1996); U. van Kolck, ibid. 49, 2932 (1994).

[6] G. P. Lepage, nucl-th/9706029.

[7] T. Park et al., Nucl. Phys. A646, 83 (1999).

[8] D. B. Kaplan, M. J. Savage, and M. B. Wise, Nucl. Phys. B478, 629 (1996).

[9] W. E. Caswell and G. P. Lepage, Phys. Lett. 167B, 437 (1986); G. T. Bodwin, E. Braaten, and G. P. Lepage, Phys. Rev. D 51, 1125 (1995); 55, 5853(E) (1997).

[10] J. V. Steele and R. J. Furnstahl, Nucl. Phys. A637, 46 (1998).

[11] T. D. Cohen and J. M. Hansen, Phys. Lett. B 440, 233 (1998).

[12] M. Luke and A. Manohar, Phys. Rev. D 55, 4129 (1997).

[13] M. J. Savage, nucl-th/9808034.

$$
\begin{aligned}
\delta^{n *, \mathrm{uv}} C_{0} & =\left(\frac{-M}{4 \pi}\right)^{n} n(n+1)\left(C_{0}^{\text {finite }}\right)^{n} C_{2}^{\text {finite }}\left(-\frac{2 \Lambda}{\pi}\right)^{n-1} \frac{\Lambda^{3}}{3 \pi}, \\
\delta^{n, \mathrm{uv}} C_{2} & =-\left(\frac{-M}{4 \pi}\right)^{n}(n+1)\left(C_{0}^{\text {finite }}\right)^{n} C_{2}^{\text {finite }}\left(-\frac{2 \Lambda}{\pi}\right)^{n} .
\end{aligned}
$$

The finite counterterms are the same as in Eq. (3.4). Thus the running couplings and amplitudes with a cutoff are the same as found using dimensional regularization.

\section{APPENDIX B: RELATIONS BETWEEN COUPLINGS IN THE OS AND PDS SCHEMES}

Here we give explicit relations between the coupling constants that occur at order $Q^{0}$ in the OS and PDS schemes; couplings on the left are in the PDS scheme, while those on the right are in the OS scheme:

$$
\begin{gathered}
C_{0}\left(\mu_{R}\right)=C_{0}\left(\mu_{R}\right), \\
\frac{C_{2}\left(\mu_{R}\right)}{C_{0}\left(\mu_{R}\right)^{2}}=\frac{C_{2}\left(\mu_{R}\right)}{C_{0}\left(\mu_{R}\right)^{2}}+\frac{g_{A}^{2}}{2 f^{2}} \frac{1}{\mu_{R}^{2}}\left[\frac{1}{C_{0}\left(\mu_{R}\right)}+\frac{M \mu_{R}}{4 \pi}\right]^{2} \\
\frac{D_{2}\left(\mu_{R}\right)}{C_{0}\left(\mu_{R}\right)^{2}}-\frac{M}{8 \pi}\left(\frac{M g_{A}^{2}}{8 \pi f^{2}}\right)=\frac{D_{2}\left(\mu_{R}\right)}{C_{0}\left(\mu_{R}\right)^{2}} \\
\frac{C_{0}^{\mathrm{p}}\left(\mu_{R}\right)}{C_{0}\left(\mu_{R}\right)^{2}}+\frac{g_{A}^{2}}{2 f^{2}} \frac{1}{C_{0}\left(\mu_{R}\right)^{2}} \\
=\frac{C_{0}^{\mathrm{p}}\left(\mu_{R}\right)}{C_{0}\left(\mu_{R}\right)^{2}}+\frac{g_{A}^{2}}{2 f^{2}}\left[\frac{1}{C_{0}\left(\mu_{R}\right)}+\frac{M \mu_{R}}{4 \pi}\right]^{2}
\end{gathered}
$$

As in Sec. VI the superscript "np" on $C_{0}\left(\mu_{R}\right)$ has been dropped.

[14] J. Gegelia, nucl-th/9806028.

[15] J. V. Steele and R. J. Furnstahl, Nucl. Phys. A645, 439 (1999).

[16] T. D. Cohen and J. M. Hansen, Phys. Rev. C 59, 13 (1999).

[17] T. Mehen and I. Stewart, Phys. Lett. B 445, 378 (1999).

[18] J. Gegelia, nucl-th/9802038.

[19] M. J. Savage, Phys. Rev. C 55, 2185 (1997).

[20] H. Georgi and A. Manohar, Nucl. Phys. B234, 189 (1984); H. Georgi, Phys. Lett. B 298, 187 (1993); Weak Interactions and Modern Particle Theory (Benjamin/Cummings, Reading, MA, 1984).

[21] D. B. Kaplan, M. J. Savage, and M. B. Wise, nucl-th/9804032.

[22] J. C. Collins, Renormalization (Cambridge University Press, Cambridge, England, 1984).

[23] M. Luke and M. J. Savage, Phys. Rev. D 57, 413 (1998).

[24] J. Chen et al., Nucl. Phys. A644, 221 (1998); M. Savage et al., ibid. A644, 235 (1998); D. Kaplan et al., Phys. Lett. B 449, 1 (1999); J. Chen et al., Nucl. Phys. A644, 245 (1998).

[25] V. G. J. Stoks et al., Phys. Rev. C 48, 792 (1993); V. G. J. Stoks et al., ibid. 49, 2950 (1994); nucl-th/9406039. (cf. http:// nn-online.sci.kun.nl/NN/)

[26] V. G. J. Stoks et al., International Symposium on the Deuteron, Dubna, Russia, 1995, nucl-th/9509032. 